\title{
Minimum Movement Relocation Strategies for Barrier Coverage on a Line Segment
}

\author{
by
}

Tamanna Zulhasnine

A thesis submitted to the Faculty of Graduate and Postdoctoral Affairs in partial fulfillment of the requirements for the degree of Master of Computer Science

\author{
Ottawa-Carleton Institute for Computer Science \\ School of Computer Science \\ Carleton University \\ Ottawa, Ontario, Canada, K1S 5B6
}

February 2013

(C) 2013, Tamanna Zulhasnine 
Library and Archives

Canada

Published Heritage

Branch

395 Wellington Street

Ottawa ON K1A ON4

Canada
Bibliothèque et

Archives Canada

Direction du

Patrimoine de l'édition

395 , rue Wellington

Ottawa ON K1A ON4

Canada
Your file Votre référence

ISBN: 978-0-494-94636-7

Our file Notre référence

ISBN: 978-0-494-94636-7
NOTICE:

The author has granted a nonexclusive license allowing Library and Archives Canada to reproduce, publish, archive, preserve, conserve, communicate to the public by telecommunication or on the Internet, loan, distrbute and sell theses worldwide, for commercial or noncommercial purposes, in microform, paper, electronic and/or any other formats.

The author retains copyright ownership and moral rights in this thesis. Neither the thesis nor substantial extracts from it may be printed or otherwise reproduced without the author's permission.
AVIS:

L'auteur a accordé une licence non exclusive permettant à la Bibliothèque et Archives Canada de reproduire, publier, archiver, sauvegarder, conserver, transmettre au public par télécommunication ou par l'Internet, prêter, distribuer et vendre des thèses partout dans le monde, à des fins commerciales ou autres, sur support microforme, papier, électronique et/ou autres formats.

L'auteur conserve la propriété du droit d'auteur et des droits moraux qui protege cette thèse. $\mathrm{Ni}$ la thèse ni des extraits substantiels de celle-ci ne doivent être imprimés ou autrement reproduits sans son autorisation.
In compliance with the Canadian Privacy Act some supporting forms may have been removed from this thesis.

While these forms may be included in the document page count, their removal does not represent any loss of content from the thesis.
Conformément à la loi canadienne sur la protection de la vie privée, quelques formulaires secondaires ont été enlevés de cette thèse.

Bien que ces formulaires aient inclus dans la pagination, il n'y aura aucun contenu manquant. 


\section{Abstract}

Mobile sensor network is a cost effective and powerful tool for numerous applications including border protection and combat field surveillance. Barrier coverage is a critical issue that can provide protection with minimal number of planted sensors by enclosing only, the border. Initially sensors are planted randomly along a line. There exists gaps if every point of the line segment is not within the sensing range of at least one sensor. It is important to exploit sensor mobility to improve barrier coverage. However it is challenging to compute the desired location that offers coverage improvement while conserving energy due to sensor movement. Given a set of sensors in random initial positions, we study the problem of computing final positions to maximize the barrier coverage of a line segment with minimal movement of sensors. We formulate the problem as linear programming framework with two variants of the objective functions: 'MinSum' that minimizes the sum of sensor movements, and 'MinMax' that minimizes the maximum distance traversed among all sensors. If the total sensing ranges of all sensors is greater than the length of the line segment, then complete coverage is possible. If the total sensing ranges of all sensors is less than the length of the line, then two different types of maximal coverage is possible: noncontiguous and contiguous. We study the same problem for variable sensing range. We also present a computationally efficient heuristic algorithm for variable range 'MinSum' problem. We demonstrate the effectiveness of these formulations on a variety of examples and provide comparisons with alternative strategies through extensive simulations. 


\section{Acknowledgements}

This thesis would have been impossible without the support and encouragement I received from several people. First and foremost I am deeply grateful to my supervisor Prof. Kranakis. His continuous guidance and keen insights inspired me to approach compelling research problems and revived my energy to complete this thesis. I am thankful to my colleagues Oscar, Gimer, and Melody for sharing their experiences and thoughts on various aspects of the scientific research. I would like to express gratitude to my committee members: Prof. Nayak and Prof. Nussbaum for their efforts and valuable feedbacks. Finally I like to thank my husband and parents who constantly believe in my efforts and provided me a 'writing-space' and specially my little daughter Megh for being inspiration in my life. 


\section{Contents}

Abstract $\quad$ iii

Acknowledgement $\quad$ iv

List of Figures viii

List of Tables $\quad$ xi

1 Introduction 1

1.1 Applications . . . . . . . . . . . . . . . . . . . . . 3

1.2 Motivation ...................... 5

1.3 Outline of the thesis ................ 6

2 Literature Review $\quad 8$

2.1 State of art of wireless sensor network . . . . . . . . . . . . 8

2.2 Cost of sensor deployment . . . . . . . . . . . . . . . . . . . 9

2.3 Critically dense sensor deployment . . . . . . . . . . . . . . . 10

2.4 Network coverage type . . . . . . . . . . . . . . . 11

2.5 Area coverage . . . . . . . . . . . . . . . . . . . . . . . 12

2.6 Barrier coverage . . . . . . . . . . . . . . . . . . 14

2.6.1 Weak barrier coverage .............. 16

2.6.2 Strong barrier coverage . . . . . . . . . . . . . . . 17

2.6.3 Sensor scheduling in barrier coverage . . . . . . . . . 18

2.6.4 Barrier coverage of a planar region . . . . . . . . . . . . 19 
2.7 Barrier coverage of a line segment . . . . . . . . . . . . 21

2.7.1 Distributed line segment barrier coverage . . . . . . . . . . . . 22

2.7.2 Energy aware line segment barrier coverage . . . . . . . . . 23

2.7.3 Optimal movement barrier coverage . . . . . . . . . . . 25

3 Optimum Movement Barrier Coverage of a Line Segment 27

3.1 Network model . . . . . . . . . . . . . . . . . 27

3.2 'MinSum' linear programming formulations . . . . . . . . . . . . . 28

3.2.1 Ordering property of optimal configuration . . . . . . . . . 29

3.2 .2 'MinSum' LP formulation for $R>L \ldots \ldots 31$

3.2.3 'MinSum' LP formulations for $R<L \ldots \ldots 34$

3.2.4 Linearized 'MinSum' LP formulation . . . . . . . . . . . . 37

3.2.5 When initial positions are not necessarily inside $[0, L] \ldots 38$

3.3 'MinMax' linear programming formulation . . . . . . . . . . . . . . 39

3.4 Simulation . . . . . . . . . . . . . . . . 40

3.4.1 Time complexity of LP and algorithmic approach . . . . . . 40

3.4.2 The impact of contiguity requirement . . . . . . . . . . . 41

4 Line Segment Barrier Coverage with Diminishing Sensing Range 47

4.1 Modified network model . . . . . . . . . . . . . . . . . . 47

4.2 Variable range 'MinSum' linear programming formulations . . . . . . 48

4.2.1 Variable range complete coverage 'MinSum' LP formulation $(R>L) \ldots \ldots \ldots \ldots$

4.2.2 Variable range maximal coverage 'MinSum' LP formulations $(R<L) \ldots \ldots \ldots \ldots \ldots$

4.2.3 Linearized 'MinSum' LP formulation . . . . . . . . . . . . . . 53 
4.3 Variable range 'MinMax' linear programming formulation . . . . . . . 54

4.4 Variable range 'MinSum' heuristic algorithm . . . . . . . . . . . . 55

4.5 Simulation . . . . . . . . . . . . . . . . . 61

4.5.1 Time complexity of variable range LP technique . . . . . . 61

4.5.2 Comparison between LP technique and heuristic method . . . 63

5 Conclusion and Future Research 66

5.1 Summary of the work . . . . . . . . . . . . . . 66

5.2 Open problem and future work ............... 68

$\begin{array}{ll}\text { References } & 71\end{array}$ 


\section{List of Figures}

2.1 Barrier Coverage . . . . . . . . . . . . . . . . . 12

2.2 Weak barrier coverage . . . . . . . . . . . . . . 16

2.3 Strong barrier coverage . . . . . . . . . . . . . . . . . 17

2.4 The sensors form a barrier by moving on the perimeter of the polygon. 20

2.5 Sensors' position. . . . . . . . . . . . . . . . . . . . . 24

3.1 An instance of complete coverage $(R>L)$ for the line segment $[0, L]$ employing 'MinSum' LP technique with nonidentical sensing range. The number of sensors is 10 . The top and bottom line segments show the initial and final positions of the sensors with their sensing rages. .

3.2 An instance of noncontiguous coverage $(R<L)$ for the line segment $[0, L]$ employing 'MinSum' LP technique with nonidentical sensing range. The number of sensors is 10 . The top and bottom line segments show the initial and final positions of the sensors with their sensing rages. . . . . . . . . . . . . . . . .

3.3 An illustration of contiguous coverage $(R<L)$ for the line segment $[0, L]$ employing 'MinSum' LP technique with nonidentical sensing range. The number of sensors is 10. The top and bottom line segments show the initial and final positions of the sensors with their sensing rages.

3.4 A comparison between LP and algorithmic approaches in terms of time complexity with complete coverage $(R>L) \ldots$. . . . . . . . . 
3.5 A comparison between LP and algorithmic approaches in terms of time complexity with noncontiguous maximal coverage (when $R<L$ and more than one gaps are possible) . . . . . . . . . . . . .

3.6 A comparison between LP and algorithmic approaches in terms of time complexity with noncontiguous maximal coverage (when $R<L$ and only one gap is possible) . . . . . . . . . . . . . . . .

3.7 The difference between noncontiguous vs contiguous LP techniques in terms of sum distance traversed. . . . . . . . . . . . . . . .

4.1 An instance of complete coverage $(R>L)$ for the line segment $[0, L]$ employing 'MinSum' LP technique with distant depended sensing range. The number of sensors is 10 . The top and bottom line segments show the initial and final positions of the sensors with their sensing rages. . 50

4.2 An instance of noncontiguous coverage $(R<L)$ for the line segment $[0, L]$ employing 'MinSum' LP technique with variable sensing range. The number of sensors is 10 . The top and bottom line segments show the initial and final positions of the sensors with their sensing rages. .

4.3 An illustration of contiguous coverage $(R<L)$ for the line segment $[0, L]$ employing 'MinSum' LP technique with variable sensing range. The number of sensors is 10 . The top and bottom line segments show the initial and final positions of the sensors with their sensing rages. . 53

4.4 An instance of right shift. Here $p=3$, therefore $j=i-p+1=i-2 . \quad 57$

4.5 An instance of left shift. Here $q=4$, therefore $k=i+q-1=i+3$. . 59

4.6 A comparison of execution time of different LP techniques. The proportional constant $\tau=0.1 \ldots \ldots \ldots \ldots$ 
4.7 A comparison between LP technique and heuristic algorithm in terms of time. The proportional constant $\tau=0.1 \ldots$. . . . . . . . . . 64 


\section{List of Tables}

2.1 The time complexity of 'MinMax' and 'MinSum' problems when $n$ sensors with identical ranges are forming a barrier of length $L$. The sum of the sensing ranges is $R . \ldots \ldots \ldots \ldots$

3.1 A comparison of time complexity between LP and algorithmic approaches with complete coverage $(R>L)$. Both 'MinSum' and 'MinMax' versions of the objective functions are considered. . . . . . . .

3.2 A comparison of time complexity between LP and algorithmic approaches with noncontiguous maximal coverage (when $R<L$ and more than one gaps are possible). Both 'MinSum' and 'MinMax' versions of the objective functions are considered. . . . . . . . . . . . .

3.3 A comparison of time complexity between LP and algorithmic approaches with contiguous maximal coverage (when $R<L$ ) and only one gap is possible. Both 'MinSum' and 'MinMax' versions of the objective functions are considered. . . . . . . . . . . .

3.4 The difference between noncontiguous vs contiguous 'MinSum' LP techniques in terms of sum distance traversed. . . . . . . . . . . . . 45

3.5 The difference between noncontiguous vs contiguous 'MinMax' LP techniques in terms of sum distance traversed. . . . . . . . . . . 
4.1 A comparison of execution time of different LP techniques to improve the coverage with minimal movement of sensors. Both 'MinSum' and 'MinMax' versions of the objective functions are considered. The proportional constant $\tau=0.1 \ldots \ldots \ldots \ldots$

4.2 A comparison between LP technique and heuristic algorithm in terms of coverage, execution time, and sum of distance traversed when $R>L$. The proportional constant $\tau=0.1 \ldots \ldots 63$

4.3 A comparison between LP technique and heuristic algorithm in terms of coverage, execution time, and sum of distance traversed when $R<L$. The proportional constant $\tau=0.1 \ldots \ldots 64$ 


\section{Chapter 1}

\section{Introduction}

Recently, sensor devices have attracted enormous attention in the field of wireless network. A sensor device consists of a sensor unit, a micro controller unit, a communication unit, a memory unit, and a power supply. It produces not only sensory data but also collects, stores, processes and delivers it. Sensors are like interfaces between the physical world and electrical devices. They help people to understand how to control and monitor machines and environments. Sensors can measure almost all kinds of physical stimulus [1]. They respond to physical stimulus such as heat, light, sound, pressure, magnetism, etc. and convert it into electrical or mechanical signals. Sensors usually come in many types and shapes depending on the amount of sensory data needed for applications.

Modern day's sensor devices are equipped with transceivers and are able to form an ad-hoc wireless sensor network (WSN) quickly. The transceiver consists of a transmitter, a receiver, and all other units necessary to perform data processing and communicate with each other over short distances. Wired network often is not feasible due to various environmental and economic conditions. Wireless sensors give relief from this messy wired network. These sensors are cost-effective with limited computation and communication power. Therefore, sensors are becoming available for monitoring the external environments. Sensors are often able to move limited distance. However, there are some severe resource constraints associated with these 
sensors, such as limited memory, low battery power, signal processing, and computation and communication abilities. Specially a single sensor can sense only a small portion of the environment. However, a group of sensors can build a larger sensing range to cover a vast region by communicating with each other efficiently.

Low-deployment cost make mobile sensors the most attractive option for deploying a wireless sensor network. There are two basic methods of sensor deployment: deterministic and random. In case of the deterministic approach, sensors are deployed in predetermined places to cover the given region. Usually it is a manual deployment method. Deterministic sensor placement can greatly minimize the number of sensors as they can be placed on the optimal positions to serve the desired purpose. However due to large geographic area and many other practical constraints, it is often not feasible to place the sensors manually. Random deployment method is more feasible for these circumstances. In random deployment technique, sensors are placed randomly in the desired region (for example in a remote or dangerous field) by dropping them from an aircraft. This is the most feasible strategy that has been vastly used in practical situation. Since the sensors are randomly deployed in a targeted area, it is difficult to find a random deployment strategy that minimizes the number of sensors maintaining sufficient coverage, low-computation and communication overhead. There is another type of deployment method which is called automatic. Here sensors are placed randomly in the initial deployment and after that they relocate themselves to the desired position in a self organizing manner. The prior knowledge about location is important here to calculate the movement to the new location. We also note that although there are many interesting questions concerning mobility models for the sensors, these will not be considered in this thesis.

When sensors make decisions locally by communicating with neighbors to solve 
a problem, the approach is distributive in nature. Such self organizing ability of the distributive approach offers reduced network load. The distributive approach therefore scales to network size. In a centralized approach, all the sensors communicate to the central system. The central system makes decisions and then informs the sensors. The working load for the central system could be high as network size grows and therefore this approach is not scalable. However sensors have limited energy, and data processing ability. In distributive approach, the number of messages required per sensor could be very high. Also decision made with local information is always suboptimal. Specially in border protection and combat field surveillance, sensors need to inform the centralized system whenever there is any intrusions. In this work we approach the problem by considering only the centralized method.

In the subsequent Sec. 1.1, we discuss a wide range of applications of the WSN. One major emerging application of WSN is to provide coverage to detect intruder in a targeted area. Coverage problem mostly depends on the coverage model of the individual sensor and the deployment method of the sensor nodes. Sensor coverage model indicates the sensing range which is usually modeled by unit disk graph when the sensors are identical. Sensors may have identical ranges or heterogenous ranges. Identical ranges means all the sensors have same sensing ranges whereas heterogenous ranges means sensors have different sensing ranges. The thesis will focus on the WSN with sensors having both identical and heterogeneous ranges.

\subsection{Applications}

There are many important applications of the WSN in practical scenarios. The literatures in [2-10] studied a wide range of potential applications of WSNs. The WSN 
has many usages in the field of environment [2-5]. A WSN can monitor the spread of forest fire, and condition of flood, and also observe bio-diversity, and environmental conditions for precision agriculture. Wireless sensors can be used near the crater of a volcano to measure temperature, pressure, and seismic activities, and studying volcanoes' interior structure [2]. The WSN also has applications in wildlife habitat monitoring such as birds and animal $[3,4]$.

There are military applications of WSNs such as military command, control, communications, and computing systems. In international borders, critical terrains, routes, paths, and straits, sensors can detect illegal intrusions and identify enemy troops and vehicles $[6,7]$. The authors in $[6]$ presented few military projects that detect, classify and track vehicles employing WSNs. The work in [7] described how sensor networks can detect and locate a sniper shooter. Another important application of wireless sensors is in the military minefield.

Smart home is another example of the application of the WSN. Sensor networks are in use for security surveillance in home and offices. Vacuum cleaners, micro-wave woven, refrigerators and other appliances, equipped with sensors, are controllable locally and remotely through the Internet. The health care system nowadays uses wireless sensors to monitor patient's body temperature, pulse rate, oxygen and glucose level in blood and other physical and biochemical parameters $[8,9]$.

Sensor networks also have usages in monitoring the structural condition of building, bridges, and roads [10]. The WSN can provide timely and accurate information about cracks and damages of buildings. Wireless sensors can also find the potential leakage on both sides of a gas and water pipeline etc. The WSN has even greater impact in industries. For example, wireless sensors are in use in nuclear industries for detecting radiation of spills. In manufacture automation, wireless sensors can read 
gas, water and electric meters, control light and smoke, and detect noxious gas. In chemical industries, sensors may detect the spread of lethal chemicals around the factory.

\subsection{Motivation}

A WSN can monitor a territory, and detect intrusions in a region. Among different types of coverage, barrier coverage is the most attractive. Barrier coverage can guaranty the coverage with minimal number of planted sensors by enclosing only the border. There are several challenges that need to be considered in designing the surveillance system through barrier coverage. Researchers have proposed two different approaches to achieve complete barrier coverage. One of the approaches considers static sensor nodes [11-15]. To ensure complete coverage, the static approach needs to place the sensors densely around the boundary. The major issue of the static deployment is to find whether the coverage is complete with the randomly deployed sensors. If a complete coverage is not possible, how many sensors are required to achieve a desired probability. In case of redundant deployment, static deployment finds the set of active sensors sufficient to provide a complete coverage. However this approach causes significant waste of sensors as it needs many redundant sensors. The mobile deployment approach can improve the initial coverage by moving the sensor to the optimal location. However sensors consume energy due to movement. In almost all cases, it is not feasible to replace batteries of the sensors once planted. Therefore it is crucial to consume energy wisely and efficiently. The minimization of movement can save energy supply. Conserving the energy due to motion is therefore a critical challenge in mobile sensor networks (MSNs) [16-22]. The deterministic deployment 
of sensors is not cost effective. However random deployment method poses challenges on the energy aware mobility control. Most of the researchers proposed different optimization algorithm as solutions to movement control strategy in MSN. All the designs are targeting to answer the following question:" Can the WSN provide a guaranteed level of coverage at final positions with minimum movement?". However most of work assumed that sensors have static or identical sensing range. Because of the optimizing goal with several constraints, we believe that the linear programming (LP) technique is suitable to formulate the optimal relocation problem with a guaranteed coverage.

In this thesis work, we focus on investigating the answer for the following question: Given a fixed number of sensors planted at random locations, determine the optimal relocations that achieve complete coverage (when possible) with minimum movement of sensors. In case a complete coverage is not possible, the objective is to maximize the coverage as much as possible. We consider this problem in one-dimensional environment where sensors are deployed in a line segment $[0 ; \mathrm{L}]$.

\subsection{Outline of the thesis}

We organize the remaining parts of the thesis as follows: Chapter 2 reviews literatures relevant to my thesis. It presents current research works and state of art of the MSN on the context of coverage. Chapter 3 presents the barrier coverage problem on a line segment with static sensing range. This chapter also proposes a LP based optimization framework to solve the problem by relocating the sensors at optimum positions with guaranteed level of coverage. Chapter 4 describes the same barrier coverage problem on a line segment with distant dependent sensing range and formulates the problem as LP that achieves guaranteed coverage with minimal movement. This chapter also 
manifests a computationally efficient heuristic algorithm to solve the same problem. Chapter 5 concludes the work and provides useful insights to design an effective line segment barrier. It also discusses some future works and open problems. 


\section{Chapter 2}

\section{Literature Review}

There has been considerable research on WSNs for the purpose of covering and monitoring a territory, and detecting intrusions in a region. We provide a greater weight on the barrier coverage of the line segment because of the focus of our thesis topic.

\subsection{State of art of wireless sensor network}

There are several foundational challenges that need to be considered in designing the surveillance system through WSNs [23]. Major foundational issues are: optimal deployment pattern, critical conditions, coverage status determination, coverage restoration, and optimal sleep wake-up. The problem of finding 'optimal deployment pattern' is to design a deployment pattern with minimum number of sensors. The deterministic deployment method is useful when sensors are expensive and their position significantly affect the application. We reckon this method is more appropriate for static sensors with indoor applications. The random deployment strategy wastes many redundant sensors without having a certain contribution to the surveillance system. However in most real scenarios with a large region, it is difficult to deploy sensors manually due to the region scalability. The scope of 'critical conditions' is to derive the conditions which minimize the number of sensors in probabilistic deployments. The problem of 'coverage status determination' is to determine the coverage efficiency of a given region. Sensors are often deviating from their locations because 
of mechanical inaccuracies, the wind, terrain characteristics, and other environmental factors. The 'coverage restoration' problem determines the additional number of sensors with their locations to restore the desired quality of monitoring within a given region. The problem of finding 'optimal sleep wake-up' is to schedule the sensors in a way that maximizes the network lifetime and confirms the best use of the resources.

MSN is a specific type of a WSN where mobility poses additional challenges in designing an efficient surveillance system. Typically mobile sensors are low battery devices and therefore energy preservation is an important issue for designing efficient WSNs. Also these mobile sensors have limited amount of memory and low computing powers. The movement of sensors consumes a large amount of energy which affects the network lifetime. The calculation of the final positions adds an extra computational overhead as well. In addition to that mobile sensors cannot maintain a routing table which makes information dissemination difficult. Sensors usually have different communication ranges as manufactured by the companies. The range depends on the transmitter and the receiver installed in the sensor device. Being a low energy device, the transmission power is also limited.

\subsection{Cost of sensor deployment}

Guarantying the coverage with minimal number of planted sensors would certainly reduce the cost of deployment. In [24], Clouqueur et al. studied the problem of deploying the least number of sensors in a targeted region that can detect intruders with a high probability. The authors suggested that the sequential deployment strategy minimizes the cost by selecting an appropriate number of sensors while still achieves 
the desired detection performance. Dhillon and Chakrabarty [25] proposed an iterative greedy heuristic algorithm using a grid approximation of deployment region to ensure the coverage goal with the least number of sensors. In [26], Pompili et al. proposed a triangular grid based algorithm that achieves maximal coverage with the least number of sensors. The authors showed that $100 \%$ coverage is possible if $d=\sqrt{3} r$ where $d$ is the distance between two adjacent sensors and $r$ is the sensing range. The authors in [27] proposed an algorithm for finding the optimal positions of sensors in a strongly connected network that gains the maximal coverage using the least number of sensors. Mehrandish et al. [28] considered sensors lying on the line segment to find the minimum number of sensors to form a barrier coverage. They proved that there is a polynomial time algorithm for identical range sensors. However, with non-identical range sensors, the problem becomes NP-hard. They also considered the barrier coverage problem for multiple line segments and circular barriers.

\subsection{Critically dense sensor deployment}

Sensor deployment in a large target area is expensive. The critical density of sensor deployment strategy plans minimum number of sensors while still guarantees the coverage. When the number of sensors is smaller than the critical density, a crossing path always exists with a high probability which means it is weak-barrier covered. Several authors have conducted research to find a desired value for the critical sensor density for the practical deployment of sensors $[11,12,29]$. Balister et al. [11] derived a reliable estimate for ensuring a global barrier coverage in a random deployment. If there is a break in the connectivity, then there exists crossing paths. The authors showed that the break occurs approximately according to the Poisson distribution. 
In [12], Chen, Lai, and Xuan analytically estimated the sensor network density that is critical for achieving a barrier coverage and connectivity or measuring the quality of the barrier coverage. In [29], Liu and Towsley studied the full coverage in one dimension and barrier coverage of two dimensional planes. The authors derived the critical threshold for the existence of a giant cluster of overlapping sensing disks using percolation theory. They concluded that there exists no crossing path beyond this critical threshold. They also defined the probability that an intruder could be detected when crossing a belt.

\subsection{Network coverage type}

There are several types of network coverage: point, area and barrier. The work in [30] studied different coverage problems in the WSN. Point coverage is one of them. It covers a particular point of interest among a large environment. Area coverage is crucial in the WSN. It provides full coverage for a particular area. Perimeter coverage is another type that provides coverage of a small portion of a large target area. Sweep coverage provides a coverage by moving a number of sensors across a sensing region while making a trade off between maximizing the detection rate of events and minimizing the number of missed detections per unit area. The detection of land mines is an example of sweep coverage [31]. Blanket Coverage achieves a deterministic deployment of sensors that maximizes the detection rate of intruders crossing in the sensing field. Barrier coverage encloses the border to identify intruders that attempt to cross the network region as shown in the Fig 2.1. Our work here focuses on the barrier coverage. 


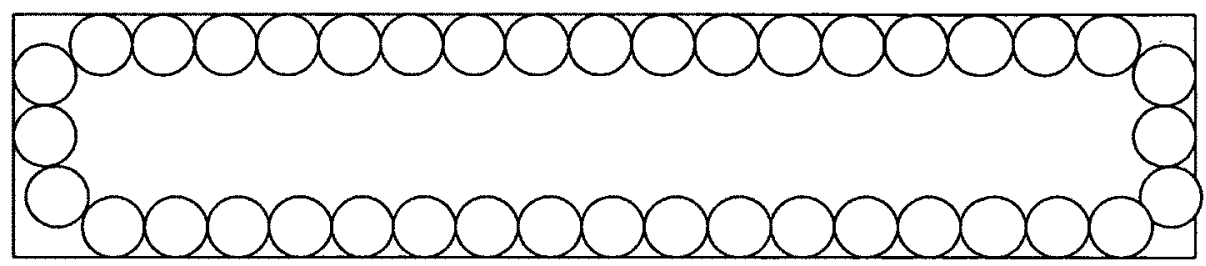

Figure 2.1: Barrier Coverage

\subsection{Area coverage}

In [32], the authors proposed several distributed algorithms that determine the desired relocations of sensors to secure the coverage holes in a specific area. The first algorithm considers the sensor density to improve the coverage. In this strategy, sensors move from the densely populated areas to sparsely covered areas. If two sensors get too close to each other, they have a repulsive force between them. Although they are evenly distributed to a region, this virtual force makes them move away from each other by half of the difference between the average distance and the distance between themselves. In addition to the mutual repulsive forces, sensors that lie too close to the boundaries are also pushed by forces to remain inside the Voronoi polygon [33]. However, if the movement to the new location causes a larger hole than the gap intended to cover, sensor's movement is restricted. In this situation, a sensor moves to the midpoint position between its target location and the new location. Therefore the notion of this algorithm is to push sensors away from the densely populated area. The second algorithm applies a greedy search to eliminate coverage holes by moving the sensors to new locations. The strategy performs a greedy search to find the local maximum coverage holes. Then the strategy moves a sensor towards the locations of its local maximum coverage hole. The amount of movement is equal to 
sensing range towards its farthest Voronoi vertex. Due to limitation of the communication range, the maximum moving distance is half of the communication radius. The movement of sensors are controlled by a movement adjustment scheme that prohibits oscillations in consecutive rounds. The authors also provided an improved version of the second algorithm. In this strategy, sensors move more conservatively towards holes such that no new holes are generated. They also evaluated their protocols with different aspects such as coverage, deployment time, moving distance, scalability to initial deployment and communication range through simulations. Once the mobile sensors move to their desired locations, they provide improved stationary coverage. If the environment changed, the algorithms had to re-compute the sensor locations accordingly to find a new stationary configuration. An important factor which affects any distributed algorithm in a WSN is the relation between the communication range and the sensing range. Zhang and Hou [34] proved that if the communication range is at least twice the sensing range of the sensor, the connectivity of the working sensors is assured. Too large radio communication is always subject to interference. The authors suggested that if the communication range is adjustable, it should be set as twice the sensing range to assure connectivity. Rather than configuring improved stationary coverage from the final relocations of sensors, the authors in [35] studied the coverage capabilities resulted from the continuous movement of the sensors. The work calculated the area coverage dynamically so that moving sensors detect intruders undetected by a stationary network. Huang and Tseng [36] proposed an algorithm to determine whether every point in a target area is covered by at least $k$ number of sensors. The proposed algorithm requires the information of location and sensing range of each sensor to verify the desired multiple levels of coverage. 


\subsection{Barrier coverage}

Barrier coverage has been attracted significant attentions in the field of WSNs recently $[14,15,37,38]$. In many locations such as country borders, coastal lines, and boundaries of battlefields, barrier coverage is vastly used as coverage strategy that provides trusted security surveillance with the minimum cost. Barrier coverage requires a chain of sensors with the sensing range of adjacent sensors overlapping or attached to each other through the deployed region. Each independent chain of sensors is working as a border guard of a barrier to detect intruders attempting to cross the region. The strength of the network coverage is determined by the number of disjoint barriers in the network. The number of sensor barriers generally depends on the number of sensors, sensing range of each sensor, and the variance of the normally distributed random offsets [37].

There is a key difference in terms of the degree of coverage parameter between barrier coverage and full coverage. Barrier coverage has several advantages compared to the full coverage. Full coverage requires every point in the deployment region to be covered. Therefore it requires many more sensors than in the case of a barrier coverage. The number of sensors required to achieve coverage depends linearly with the width [14] of the covered region. If the width of the deployment region is three times of the sensing range, the full coverage requires more than twice the density of barrier coverage. It is more efficient than the full coverage since it requires fewer sensors for detecting intruders. The 'sleep wake up problem', which determines a sleeping schedule for sensors to maximize the network lifetime, is solvable in polynomial-time for a barrier coverage [38]. The same problem is NP-Hard for the full coverage [39]. 
Whether a barrier coverage can detect an intruder depends on a number of parameters such as the deployment pattern, and the number of crossing paths. Sensor deployment methods have a great influence on barrier coverage. The goal is to achieve full barrier coverage where sensors are placed side by side regularly in straight lines across the region. For some applications, it is possible to place sensors manually in a targeted region by aligning them in straight line. In that case, minimum number of sensors is required to provide barrier coverage. However, this is not possible for most of the applications in real life. Due to large geographic area, often sensors are deployed in regions of an irregular long belt shape. In some cases, sensors are dropped from airplanes or launched by artilleries randomly to the area. With random deployment of sensors, barrier coverage depends on the spatial distribution of sensor locations.

Barrier coverage is also related with the crossing paths an intruder takes. A crossing path crosses from one side to the other side of the width of region. If an intruder has no prior knowledge of the location of the sensor, the optimal crossing paths that minimize the probability of being detected in a two-dimensional rectangular network are the orthogonal crossing paths. In contrast to the other coverage criteria, barrier coverage needs information about its environment and also the way in which intruders will attempt to cross through the region. Although the desired region is not limited to any particular shape, the entry and exit point of the region should be defined precisely that will allow the intruders to cross. Meguerdichian et al. [40] proposed a coverage metric that measures of the quality of service offered by the WSN. The proposed polynomial-time algorithm uses the graph theory and the computational geometry to compute the worst- and best-case coverage of path between two end points. In [41], the authors proposed a centralized algorithm to measure the likelihood that an 
intruder is detected while crossing a path between two points. The work considered that the sensing abilities are less if the distance or the sensing time (exposure) is longer.

\subsubsection{Weak barrier coverage}

Barrier coverage is classified into categories: strong barrier coverage and weak barrier coverage. Weak barrier coverage guarantees to detect intruders moving along congruent paths. Figure 2.2 depicts a network of weak barrier coverage where all orthogonal

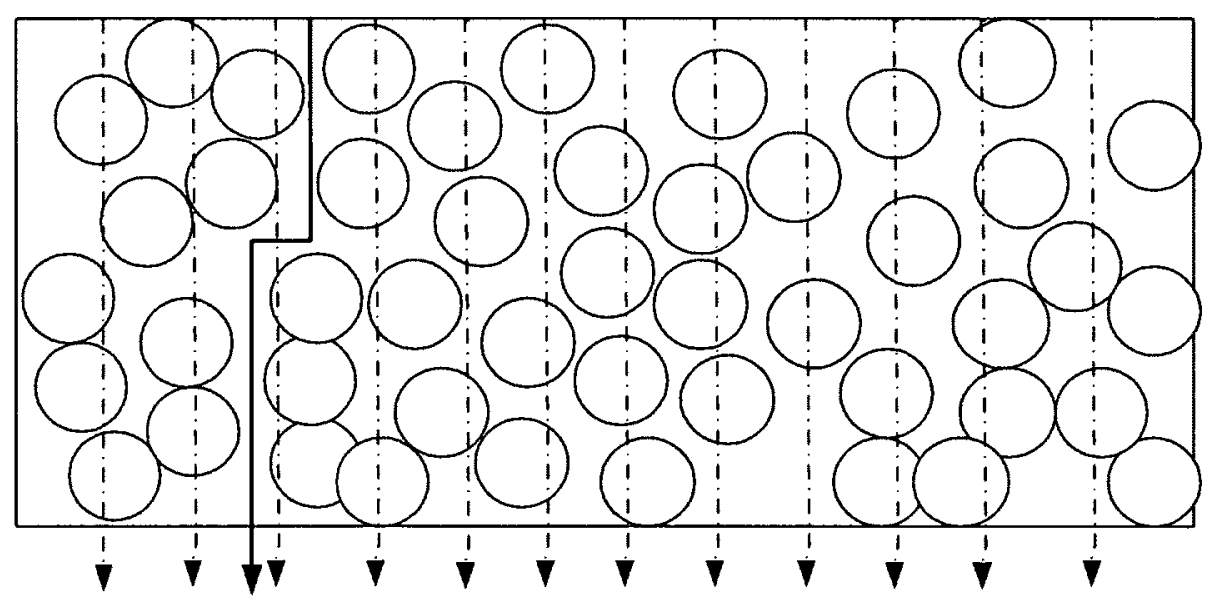

FIGURE 2.2: Weak barrier coverage

crossing paths (dashed paths) are covered and a single crossing path is (solid path) uncovered through the region. Kumar et al. [14] introduced the idea of $k$-barrier coverage for the WSN. A path is $k$-barrier covered, if the intruder cannot cross the complete width of the region without being detected by at least $k$ sensors. They proposed a centralized algorithm that calculates the critical conditions to decide whether a region is weakly $k$-barrier covered with random deployment of the sensors. The authors also provided an optimal deployment pattern to achieve $k$-barrier coverage when 
sensors are planted deterministically.

\subsubsection{Strong barrier coverage}

Strong barrier coverage does not contain any gaps. No intruders can cross the region undetected irrespective of what crossing paths they would choose. Figure 2.3 depicted strong barrier coverage provided by the WSN.

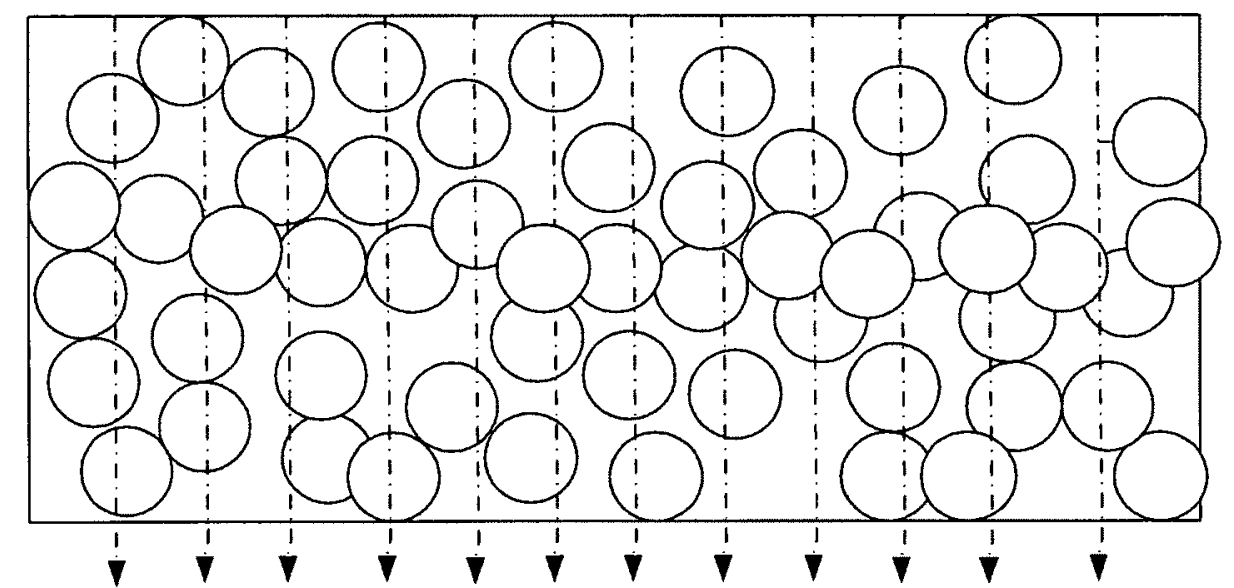

FIGURE 2.3: Strong barrier coverage

In [15], Liu et al. provided critical conditions for a strong barrier coverage when sensors are planted according to a Poisson point process. The authors presented an efficient distributed algorithm to develop multiple disjoint barriers in a large sensor network to cover a long boundary area of an irregular shape without any constraints on crossing paths. Strong barrier coverage of a sensor network depends on its widthto-length ratio. Regardless the density of sensors, if the width of the rectangular targeted region area is smaller than the logarithm of the length, then the probability of existing uncovered crossing paths tends to ' 1 '. It proves that there is no strong barrier coverage on the network. On the other hand, if the node density achieves 
a certain value and the width is asymptotically larger than the logarithm of the length, the network has a strong barrier coverage. Here the original network has been divided into a number of small segments where each segment independently computes the barrier coverage for its own area. Horizontal barriers have been constructed in each segment connected by vertical barriers of neighboring vertical strips. This approach significantly reduces delay, communication overhead, and computation costs compared to the centralized approach [14]. It also improves the robustness of the barrier coverage as it prevents intruders from moving to adjacent segments throughout the entire region. In addition, it improves the local barrier coverage since it computes barriers locally between vertical barriers at the two ends for each segment.

\subsubsection{Sensor scheduling in barrier coverage}

Numerous algorithms have been proposed recently to make the optimum use of energy $[13,38,42]$. The radio of the wireless sensor can be in one of the following four states: transmit, receive, idle and sleep. Experimental studies on power usage in [43] showed that the transmitting and receiving states and even the idle state require a significant amount of power as compared to that of the sleep state. Therefore the only way to save energy is to go to the sleep state by turning the radio off. In [44], Cardei et al. proposed a heuristic algorithm that finds $k$ disjoint sets, each capable of covering a targeted region. Sets are activated successively and only sensors from the current active set monitor the target region while remaining sensors of the other sets are in a low-energy sleep mode. As sensors work in shifts, this scheme elongates the network lifetime. Sensor activity scheduling, especially when sensors are deployed randomly, is an important task that may help to achieve prolonged network life. The 
idea is to activate sensors as few as possible alternatively while still providing a barrier coverage. The work in [13] proposed an energy efficient sensor activity scheduling algorithm that exploits collaborations among neighboring sensors to reduce the number of active sensors required to cover a barrier. Kumar et al. [38] proposed two heuristic algorithms that maximize the network lifetime while maintaining the desired quality of monitoring. Both algorithms achieve optimality in the sense that they ensure the upper bound of the network lifetime. In random independent sleeping [42] scheme, each sensor wakes up for some time with some probability $p$, independently of every other sensors. This scheme increases the network life time with a factor of $1 / p$. The scheme also does not require any central coordination or no massage exchange around any neighborhood. However, no deterministic guarantee of barrier coverage is possible. The work in [45] developed an optimization framework which finds the smallest number of sensors that must be active in order to guarantee the coverage of the same region as the complete set of sensors.

\subsubsection{Barrier coverage of a planar region}

The authors in [16] investigated the barrier coverage problem for a two dimensional space. Initially the sensors are located inside the planar region. Each sensor has prior knowledge about its geographic location. The perimeter of a convex polygon or a circle can be the barrier of a planar region. The authors proposed two algorithms that find the optimal positions of the sensors to build a barrier by moving the sensors from the inside to the perimeter of the polygon. The relocation of the sensors on the perimeter of the polygon forming a fence is optimal when either the sum of the distances traveled by all sensors or the maximum distance of any sensors is minimized. 
Two variants of the problems are named as 'MinSum' and 'MinMax' problems. The sum of coverage of the sensors is at least equal to the length of the barrier of the targeted area. When the sum of the coverage of the sensors is exactly equal to the length of the barrier, the optimal position is achieved by moving the sensors along the parameter of the circle or polygon with equidistance from each other of amount twice the sensing range. An extended version of this work is published here [17]. Fig. 2.4

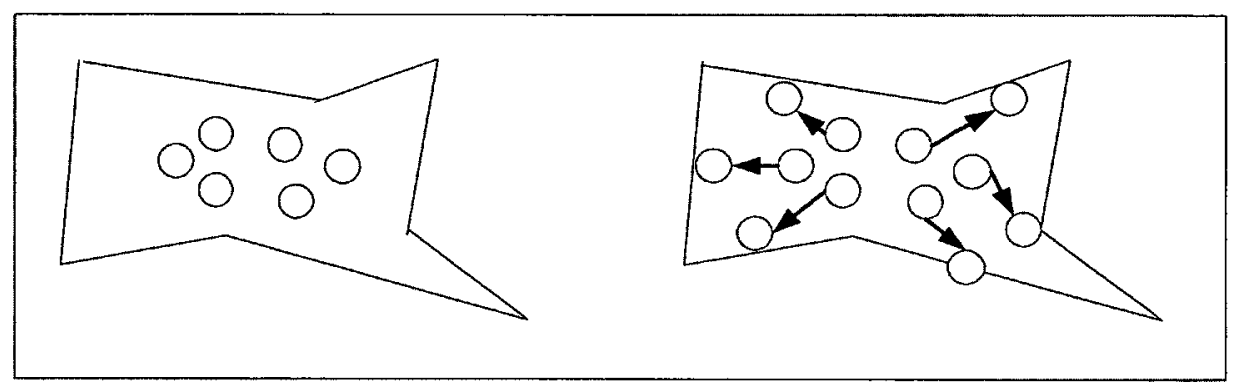

FIgURE 2.4: The sensors form a barrier by moving on the perimeter of the polygon.

illustrates how sensors move from their initial positions (left) onto the perimeter of the $n$-gon (right). The proposed 'MinSum' algorithm requires an $O\left(n^{3.5} \ln n\right)$-time and the 'MinMax' algorithm requires an $O\left(m n^{3.5} \ln n\right)$-time to find the optimal positions of the sensors. Here $n$ is the number of sensors and $m$ is the number edges of the polygon. The authors also proposed a polynomial time approximation scheme to solve the 'MinSum' problem with an approximation $1+\epsilon$ and an $O\left(\frac{1}{\epsilon} m n^{5}\right)$ running time. In [18], the authors claimed that the 'MinMax' problem is solvable within $O\left(n^{2.5} \ln n\right)$-time.

In [46], the authors proposed a distributed algorithm based on the virtual force to spread the sensors from their initial positions to the points on the convex hull of a region uniformly. The authors considered the case of random deployment of sensors and made an attempt to find an optimal distribution of sensors. The strategy involves 
two steps: boundary seeking and barrier forming. In the first step, the sensors, with no prior knowledge of the protected region, seek for the boundary autonomously. If a sensor is out of the range of the region of protection, it moves along the spiral direction to find the boundary. The spiral path provides equal probability on all directions to detect a boundary. If the region is within the sensing range of the sensor, the sensor moves directly on the boundary. If the sensor is inside the region, it takes the shortest path to be on the boundary. In the second step, the sensors apply a cooperative strategy to move automatically from their sources to destinations with only local information. If the internal angle of a sensor with its two immediate neighbors is less than 180 degree, it moves to a position that has equal distances from its both neighbors. If the internal angle a sensor with its two immediate neighbors is greater or equal to 180 degree, it moves to the midpoint between its neighbors. If a sensor has a single neighbor it moves away from its neighbor to a distance equal to twice of its sensing range. Chen et al. [47] proposed a localized algorithm that guarantees the detection of intruders whose movement is confined to a slice of the belt region. In this strategy, each sensor schedules its own activity based on the information around its neighbors.

\subsection{Barrier coverage of a line segment}

Different deployment strategies may have significantly different effects on barrier coverage. In [37], authors described a line based sensor deployment approach. For an example, if sensors are dropped from an aircraft to the target region, their distribution will follow a line with random offsets due to the wind and other environmental factors. The offset causes sensor placement errors. Previous works assumed that sensors are 
distributed randomly in a large area. The authors argued that line based deployment model is more realistic than two-dimensional uniform sensor deployment model. The authors derived a tight lower-bound that ensures the existence of barrier coverage under line-based deployments. The authors argued that line-based barrier coverage deployment outperforms the Poisson model based deployment provided offsets are relatively small compared to the sensor's sensing range.

It needs much more sensors to provide guaranteed barrier coverage with the conventional single-round sensor deployment. Sensors need to be deployed in a way where it needs to be position with small deployment interval to counter the random deployment during the sensor deployment process. In another way, sensor deployment can be divided into multiple rounds with larger deployment intervals in the previous rounds and then later round can be deployed with smaller interval to fill the coverage gaps generated in the previous rounds. With this approach, a significant number of sensors can be saved which is cost effective. Splitting sensor deployment into multiple rounds may incur higher deployment cost. The work also studied sensor deployments along multiple lines which may offer robustness through multiple lines of defense. The distance between adjacent lines and the random offsets affects the performance of barrier coverage.

\subsubsection{Distributed line segment barrier coverage}

In [48], the authors demonstrated a distributed motion coordination algorithm for mobile sensors to ensure a protected region between two landmarks or points. By employing the proposed algorithm, the mobile sensors relocate autonomously on a line segment between the two points with the same interval. This way, every point on 
the line segment is covered by the sensing region of at least one sensor. After sensor deployment, the sensors maintain this configuration so that the sensors can cover all crossing paths through the line. In real life, targeted region is generally wide, and low-cost and low-power mobile wireless sensors are used to detect any object entering in a secured region. Mobile sensors can be dispatched near the landmarks to deploy a barrier coverage. Rather than concentrating on locations and number of sensors, the authors focused on how to drive a group of mobile sensors autonomously and cooperatively to form a barrier coverage. The mobile sensors do not have prior knowledge about the targeted position that results in less computational and communication overhead. Each sensor only requires information around its closest neighbors. It is therefore a distributed and scalable approach.

\subsubsection{Energy aware line segment barrier coverage}

In [49], Shen et al. presented a centralized energy efficient algorithm that finds the optimum relocating position to provide a barrier coverage. The centralized algorithm requires the knowledge of initial positions of all sensors in advance. If mobile sensors (of sensing range $r$ ) are deployed into a straight line of length $L$, the minimum number of sensors required is $L / 2 r$ for achieving barrier coverage. All mobile sensors are aligned to a straight line in parallel with the bottom line of the given area. When the sensors are aligned into a diagonal of the given rectangle targeted region of with $W$, then the minimum number of mobile sensors required to achieve barrier coverage

is $\sqrt{\left(L^{2}+W^{2}\right) / 2 r}$. The number of sensors required in a straight line deployment pattern is fewer than that of the diagonal deployment pattern. See Fig. 2.5. However regardless of any deployment strategies, if one of the sensors gets deactivated, this 


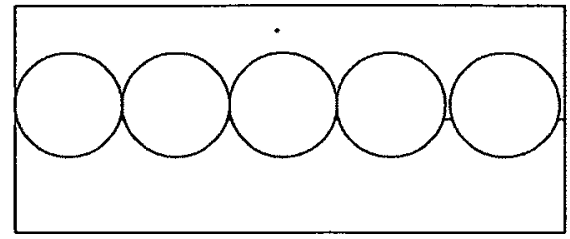

(a) The sensors are aligned to a straight line parallel to the bottom line.

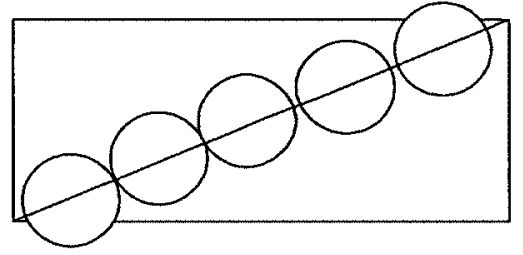

(b) Diagonal arrangement of sensors

Figure 2.5: Sensors' position.

will lead to the failure of providing barrier coverage in the network. Relocation of sensors causes decay in energy consumption. In order to save energy consumption of relocation, moving trips of sensors should be as short as possible. One way is to relocate sensor at a position close to its previous one if possible while maintaining the barrier coverage. The authors also proposed a distributed barrier algorithm that scales to the network size. Sensors that applied distributed algorithm initially store new position location instead of moving there. After deciding final position to relocate, each sensor then moved to that position. This way sensors save valuable energy. The distributed algorithm can only converge when the moving unit is below a certain threshold value. It is also challenging to design a distributed algorithm as any individual sensor cannot locally determine whether the given area is barrier covered or not. In [50] Li et al. investigated the linear coverage problem where sensors are fixed, however may adjust their sensing ranges. The authors presented an optimal range assignment algorithm that minimizes the cost of sensing energy of each sensor while still guarantying the linear coverage. Two variants of the optimization problems are: discrete and continuous power assignments. 


\subsubsection{Optimal movement barrier coverage}

The authors in [19] studied how to relocate sensors to form multiple barriers in a thin strip. They provided an algorithm to construct maximum number of barriers with minimum sensor moving distance. For each line of barrier, the strategy first computes the grid points with the coordinates $(2 i+1) r, i=0,1, \ldots, l / 2 r-1$. Then the authors applied 'minimax grid matching problem' [20] that perfectly match sensors' relocation points to the grid points. The authors also analyzed the effects of the density and proposed an algorithm that determines the presence or absence of a barrier.

TABLE 2.1: The time complexity of 'MinMax' and 'MinSum' problems when $n$ sensors with identical ranges are forming a barrier of length $L$. The sum of the sensing ranges is $R$.

\begin{tabular}{l|ll}
\hline Coverage & Contiguous & Noncontiguous \\
\hline$R<L$ & $O(n)$ & $O\left(n^{2}\right)$ \\
$R=L$ & $O(n)$ & Not applicable \\
$R>L$ & $O\left(n^{2}\right)$ & Not applicable \\
\hline
\end{tabular}

In [21], the authors investigated the problem of moving sensors along a line to provide a barrier coverage. The objective is to minimize the maximum moving distance among the sensors and the problem named as the 'MinMax' problem. The authors studied a few variants of the problem: (a) if a complete coverage is possible or not (b) when a complete coverage is not possible, whether or not a maximal coverage is required to be contiguous. If the sum of the total sensing range $(R)$ is greater than the length of line segment, a complete coverage is feasible. When sensors have identical ranges and a complete coverage is impossible, the authors gave linear time optimal algorithms that achieves maximal coverage both for the contiguous and 
non-contiguous case. With identical ranges, if a complete coverage is possible, the algorithm can solve the problem in $O\left(n^{2}\right)$ time, where $n$ is the number of the sensors. The authors also investigated approximate maximal coverage for both contiguous and non-contiguous cases. They proposed two linear time optimal algorithms that achieve maximal coverage for both the contiguous and non-contiguous cases. One of them an $O(n)$-time algorithm and the other one is a $1+\epsilon$ approximation scheme with running time $O\left(n \ln n\left(\frac{\ln \left(\frac{C}{g}\right)}{\ln (1+\epsilon)}\right)\right)$. Here $g$ is the length of the largest gap between $n$ sensors on a line segment of length $L$. The important thing to notice here that the 'MinMax' algorithm is order preserving. The solution of the algorithm keeps the original ordering of sensors in their final positions. In a subsequent work [22], the authors approached the same problem with a different optimizing technique. Rather than minimizing the maximum distance traversed by any sensor, the problem considers minimizing the sum of the distances traversed by all sensors to establish a barrier by moving the sensors to final positions. In terms of time complexity, the results of the 'MinMax' and MinSum' problems have some similarities. Table 2.1 summarizes the time complexity for 'MinMax' and 'MinSum' problems. When the sensors have nonidentical ranges, or if one of the sensors has a fixed position, the problems appear to be NP-complete. 


\section{Chapter 3}

\section{Optimum Movement Barrier Coverage of a Line Segment}

In this chapter, we study the problem of forming a barrier of a line segment. Initially sensors are planted randomly along a line. They are sorted by coordinate. There exists a gap if some points of the line segment are not within the sensing range of at least one sensor. We propose an optimization framework to find a linear segment barrier by relocating the sensors at optimum positions. We formulate the problem as LP and compare the solutions with previous algorithms proposed in $[21,22]$. We consider two variants of the objective functions: 'MinSum-LP' that minimizes the sum of sensor movements, and 'MinMax-LP' that minimizes the maximum distance traversed by a sensor among all sensors. For each variant of the objective functions, we consider three different coverage approaches. If the total sensing range from all sensors is greater than the length of the line, then a complete coverage is possible. If the total sensing range from all sensors is less than the length of the line, then two different types of maximal coverage are possible: noncontiguous and contiguous.

\subsection{Network model}

There are $n$ sensors denoted as $S_{1}, S_{2}, \ldots, S_{n}$ planted randomly on a line. We define a barrier as a closed line of interval $I=[0, L]$ with two end points 0 and $L$. The 
point $L>0$. Sensors are restricted to move with constant and identical speeds along the line towards the final positions. The initial positions of sensors are $x_{1}, x_{2}, \ldots, x_{n}$ which may be either the inside or the outside of the line $I$, and the corresponding final positions are $y_{1}, y_{2}, \ldots, y_{n}$. The sensing range of the $i$-th sensor is $r_{i}$, where $1 \leq i \leq n$. Usually this sensing range is predetermined by the manufacturer. The sensor $S_{i}$, whose position is at $x_{i}$, is defined as $I\left(S_{i}, x_{i}\right)=\left[x_{i}-r_{i}, x_{i}+r_{i}\right]$. Therefore the sensor $S_{i}$ covers an interval of length $2 r_{i}$. All the points inside this interval are considered as covered. In other words, if the Euclidean distance from any point to the center $x_{i}$ is at most $r_{i}$, then this point is considered to be covered by the sensor. A gap is a subinterval of the closed line which is not within the range of any sensors. A gap may be a semi-closed interval if one of the endpoints of the gap is either 0 or $L$. An overlap is defined as a sub-interval of $I$ which is covered by more than one sensor. One important thing to note is that the optimization technique does not enlarge any of the gaps or overlaps due to movement. Also the technique does not create any new gap or overlap. The total sensing range is the sum of lengths of covering intervals of each sensor and is denoted by $R=\sum_{i=1}^{n} 2 r_{i}$. Our goal is to fill the gaps of the line segment by moving the sensors to the optimal positions.

\section{2 'MinSum' linear programming formulations}

Here we formulate the problem of forming a line segment barrier from initial random deployment of sensors as LP. Our formulation is valid even for nonidentical ranges of sensors. When a complete coverage is possible $(R>L)$, the LP solves for the optimal final positions of sensors such that the sum of distances traversed by all sensors is minimized. When a complete coverage is not possible $(R<L)$, the LP formulation 
maximizes the coverage in two different arrangements: contiguous and noncontiguous. A contiguous arrangement of sensors attains the largest possible coverage $R$, if the provided coverage is a contiguous subinterval of $[0, L]$. A noncontiguous arrangement of sensors achieves the largest possible coverage $R$, when the provided coverage is a collection of disjoint subintervals. At first we present LP formulations which are valid when the initial positions of the sensors are inside the interval $[0, L]$.

\subsubsection{Ordering property of optimal configuration}

Here we review an important property of the optimal configuration from [21] for the completeness of my work. The authors [21] proved that final positions of the sensors in the 'MinSum' optimal solution preserve the initial ordering of sensors. This property is named as 'order preserving'. That is no two sensors never cross each other on their way to the optimal locations. The following ordering property is also valid for sensors with nonidentical sensing range as long as any sensor does not cover more (on its left side) than that of its left side sensor and vice versa. That is $x_{i}-r_{i}>x_{i-1}-r_{i-1}$ and $x_{i}+r_{i}<x_{i+1}+r_{i+1}$. We note that the LP optimization described in the sequel is stated for the more general case of arbitrary sensing ranges $r_{i}, i=1,2, \ldots, n$. However, the resulting movement of the sensors is optimal only when the sensing ranges are equal.

Lemma 3.2.1 If $x_{i} \leq x_{j}$ and $y_{i}>y_{j}$ are real numbers.

$$
\left|x_{i}-y_{i}\right|+\left|x_{j}-y_{j}\right| \geq\left|x_{i}-y_{j}\right|+\left|x_{j}-y_{i}\right|
$$

Proof Let us consider five possible arrangements depending on the relative values of 
$x_{i}, x_{j}, y_{i}, y_{j}$

$$
\begin{aligned}
& x_{i} \leq x_{j} \leq y_{j} \leq y_{i} \\
& x_{i} \leq y_{j} \leq x_{j} \leq y_{i} \\
& x_{i} \leq y_{j} \leq y_{i} \leq x_{j} \\
& y_{j} \leq x_{i} \leq y_{i} \leq x_{j} \\
& y_{j} \leq y_{i} \leq x_{i} \leq x_{j}
\end{aligned}
$$

In the first case, we have that $x_{i} \leq x_{j} \leq y_{j} \leq y_{i}$. Therefore, we derive

$$
\begin{aligned}
\left|x_{i}-y_{i}\right|+\left|x_{j}-y_{j}\right| & =\left(y_{i}-x_{i}\right)+\left(y_{j}-x_{j}\right) \\
& =\left(y_{i}-y_{j}\right)+\left(y_{j}-x_{j}\right)+\left(x_{j}-x_{i}\right)+\left(x_{i}-x_{j}\right) \\
& =\left(y_{j}-x_{i}\right)+\left(y_{i}-x_{j}\right) \\
& =\left|x_{i}-y_{j}\right|+\left|x_{j}-y_{i}\right|
\end{aligned}
$$

Therefore, the inequality in Eq. 3.1 holds. In the third case, we have that $x_{i} \leq y_{j} \leq$ $y_{i} \leq x_{j}$. Therefore, we derive

$$
\begin{aligned}
\left|x_{i}-y_{i}\right|+\left|x_{j}-y_{j}\right| & =\left(y_{i}-x_{i}\right)+\left(x_{j}-y_{j}\right) \\
& =\left(y_{i}-y_{j}\right)+\left(y_{j}-x_{i}\right)+\left(x_{j}-y_{i}\right)+\left(y_{i}-y_{j}\right) \\
& \geq\left(y_{j}-x_{i}\right)+\left(x_{j}-y_{i}\right) \\
& =\left|x_{i}-y_{j}\right|+\left|x_{j}-y_{i}\right|
\end{aligned}
$$

The inequality in Eq. 3.1 also holds for the remaining second, fourth and fifth cases 
similarly.

Corollary 3.2.2 (Order Preservation) For any of the 'MinSum' optimization problems, if $x_{1} \leq x_{2} \leq \ldots \leq x_{n}$ are the initial positions of sensors $S_{1}, S_{2}, \ldots, S_{n}$ then there exists an optimal solution of the problem such that their final destinations satisfy $y_{1} \leq y_{2} \leq \ldots \leq y_{n}$.

If $R=L$, the Corollary 3.2.2 implies that the optimal solution is straightforward. The optimal relocations are: $y_{1}=r_{1}$, and $y_{i}=\sum_{j=2}^{i-1} 2 r_{j}+r_{i}, \quad i=2, \ldots, n$. Therefore, we only consider the case when $R>L$ and $R<L$.

Proof An order preserving final positions in an optimal solution directly follows from Lemma 3.2.1.

\subsection{2 'MinSum' LP formulation for $R>L$}

When $R>L$, a complete coverage is possible. The following constraints in Eqs. $3.3,3.4$, and 3.5 along with the objective function in Eq. 3.2 constitute the LP formulation:

$$
\begin{aligned}
\operatorname{minimize} & \sum_{i=1}^{n}\left|x_{i}-y_{i}\right| \\
\text { subject to: } & y_{1} \leq r_{1} \\
& y_{i+1}-y_{i} \leq r_{i}+r_{i+1}, \quad i=1,2, \ldots, n-1 \\
& y_{n} \geq L-r_{n}
\end{aligned}
$$

The objective function in Eq. 3.2 minimizes the sum of distances traversed by all sensors. The constraints in Eq. 3.3, 3.4, and 3.5 ensure the complete coverage of the 
line segment.

Theorem 3.2.3 The 'MinSum' optimization problem of $n$ sensors, with initial positions inside the line segment $[0, L]$, is solvable using the simplex method in time $O\left(n^{2}\right)$ provided the total sensing range of the sensors is greater than the length of line segment $(R>L)$.

Proof We first make two claims to prove the theorem.

Lemma 1: The LP constraints in Eq. 3.3, 3.4, and 3.5 guarantee that $0 \leq y_{i} \leq L$, for $i=1,2, \ldots, n$ provided all sensors start at initial positions inside the interval $[0, L]$. Proof: We prove this claim by the following way. First consider the lower bound for $y_{i}$. Assume $A$ is the set of all $i$ such that $y_{i}<0$. Set $y_{i}=0$ for all $i \in A$. This modification improves the objective function stated in Eq. 3.2. Moreover, this modification does not violate any of the constraints. Similar way we observe the upper bound which completes the correctness of the claim.

lemma 2: The constraints in Eq. 3.3, 3.4, and 3.5 guarantee that $n$ sensors provide the coverage of the line segment $[0, L]$.

Proof: We can prove this by contradiction. We assume there exists a point $p \in[0, L]$ which is not covered by any sensor, such that

$$
\left|y_{i}-p\right|>r_{i}, \quad \text { for all } i=1,2, \ldots, n
$$

Consider $i$ is the largest integer such that $y_{i}<p$. If such an $i$ does not exist then it must be because of $y_{i} \geq p$, for all $i=1,2, \ldots, n$. There are three possible instances of $p$. Let us inspect the first case if $y_{1} \geq p$. Therefore, $y_{1}-p>r_{1}$ which contradicts 
the LP constraint $y_{1} \geq r_{1}$ in Eq. 3.3. Another instance for which such an $i$ could exist is $i=n$. Then it becomes $y_{n}<p$. Therefore $p-y_{n}>r_{n}$ which contradicts the LP constraint $y_{n} \geq L-r_{n}$ in Eq. 3.5. For all other instances for which such an $i$ could exist are $i<n$, and that lead to $y_{i}<p$. In this case we have $p-y_{i}>r_{i}$. Again as $p \geq y_{i+1}$ by maximality of $i$, we have $y_{i+1}-p \geq r_{i+1}$ which contradicts with the LP constraints $y_{i+1}-y_{i} \leq r_{i}+r_{i+1}$ in Eq. 3.4. To prove Theorem 3.2.3 we modify variables $\Delta_{i}=x_{i}-y_{i}$ to transform the 'MinSum' L.P problem to the following equivalent LP problem.

$$
\begin{aligned}
\operatorname{minimize} & \sum_{i=1}^{n}\left|\Delta_{i}\right| \\
\text { subject to: } & x_{1}-\Delta_{1} \leq r_{1} \\
& x_{i+1}-\Delta_{i+1}-\left(x_{i}-\Delta_{i}\right) \leq r_{i}+r_{i+1}, \quad i=1,2, \ldots, n-1 \\
& x_{n}-\Delta_{n} \geq L-r_{n}
\end{aligned}
$$

This new LP problem has $n$ variables and $n+1$ constraints. Therefore, the simplex method will have $p=\left(\begin{array}{c}n+1 \\ n\end{array}\right) \in O(n)$ pivots. As the objective function has the form 'sum of the absolute values', the derivation of the simplex solution has $O(p n)=O\left(n^{2}\right)$ time complexity [51].

Figure 3.1 depicts how sensors provide a complete coverage by moving to the final positions. There is no gap between sensors. The final positions are optimized employing LP technique such that the sum of distances traversed by all sensors is minimized. We will provide the details of the simulation method in Sec. 3.4. 


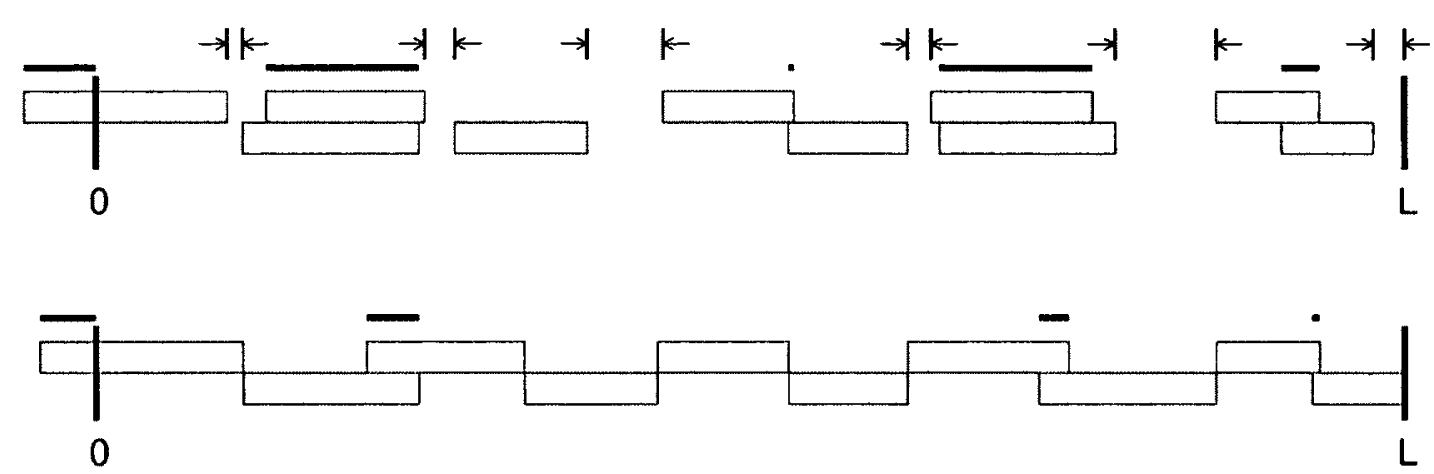

Figure 3.1: An instance of complete coverage $(R>L)$ for the line segment $[0, L]$ employing 'MinSum' LP technique with nonidentical sensing range. The number of sensors is 10 . The top and bottom line segments show the initial and final positions of the sensors with their sensing rages.

\subsection{3 'MinSum' LP formulations for $R<L$}

When a complete coverage is not possible, we present two variants of LP formulations: noncontiguous and contiguous.

\section{'MinSum' LP with noncontiguous coverage}

With $R<L$, a noncontiguous coverage requires that no overlap exists. However, gaps are allowed in this case. The Eqs. 3.6, 3.7, 3.8, and 3.9 constitute the LP formulation for noncontiguous arrangement with $R<L$.

$$
\operatorname{minimize} \sum_{i=1}^{n}\left|x_{i}-y_{i}\right|
$$

subject to: $y_{1} \geq r_{1}$

$$
\begin{aligned}
& y_{i+1}-y_{i} \geq r_{i}+r_{i+1}, \quad i=1,2, \ldots, n-1 \\
& y_{n} \leq L-r_{n}
\end{aligned}
$$


Theorem 3.2.4 The noncontiguous 'MinSum' optimization problem of $n$ sensors is solvable using the simplex method in time $O\left(n^{2}\right)$ provided the total sensing range of the sensors is less than the length of line segment $(R<L)$.

Proof The constraints in Eq. 3.8 ensure that no overlap exists between two sensors. The constraints in Eq. 3.7 and 3.9 guarantee that no unwanted coverage exists outside the line segment $[0, L]$. And optimality is ensured by Lemma 3.2.1. The noncontiguous 'MinSum' LP formulation has an equivalent formulation of the 'MinSum' LP formulation with $R>L$ except the inequalities signs in the constraints. Therefore, the noncontiguous 'MinSum' LP formulation is solvable in $O\left(n^{2}\right)$ time.

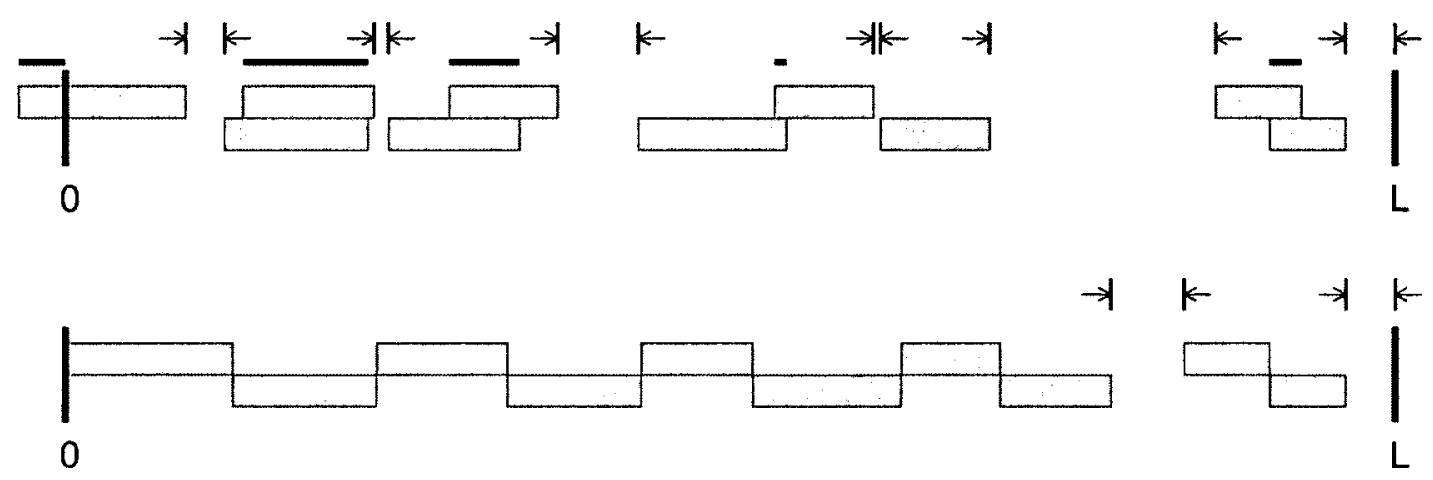

FIGURE 3.2: An instance of noncontiguous coverage $(R<L)$ for the line segment $[0, L]$ employing 'MinSum' LP technique with nonidentical sensing range. The number of sensors is 10 . The top and bottom line segments show the initial and final positions of the sensors with their sensing rages.

Figure 3.2 illustrates an example of noncontiguous coverage. Obviously there are multiple gaps due to $R<L$ and noncontiguous arrangement. However, the coverage improves $28.05 \%$ when compared to initial coverage for this particular example. 


\section{'MinSum' LP with contiguous coverage}

With $R<L$, a contiguous coverage requires that no overlap or gap exist between two adjacent sensors. If the final position of sensor $S_{1}$ is computed, the final positions of the other sensors can be computed from the equalities $y_{i+1}-y_{i}=r_{i}+r_{i+1}, \quad i=$ $1,2, \ldots, n-1$. Therefore, we remove the constraints in Eq. 3.4. The objective function in Eq. 3.2 changes as well. The Eqs. in $3.10,3.11$, and 3.12 define the LP formulation for continuous arrangement with $R<L$.

$$
\begin{array}{cl}
\operatorname{minimize} & \left|x_{1}-y_{1}\right|+\left|x_{2}-\left(y_{1}+r_{1}+r_{2}\right)\right| \\
& +\sum_{i=3}^{n}\left|x_{i}-\left(y_{1}+r_{1}+r_{i}+2 \sum_{j=2}^{i-1} r_{j}\right)\right| \\
\text { subject to: } & y_{1} \geq r_{1} \\
& y_{n} \leq L-r_{n}
\end{array}
$$

The constraints in Eq. 3.11, and 3.12 ensure that entire sensing ranges of the endmost sensors of both sides are covering the line segment.

Theorem 3.2.5 The contiguous 'MinSum' optimization problem of $n$ sensors is solvable in time $O(n)$ provided the total sensing range of the sensors is less than the length of line segment $(R<L)$.

Proof With $R<L$, the contiguous 'MinSum' optimization problem has only single variable, and two constraints. Therefore the time complexity of the contiguous 'MinSum' LP problem is $O(n)$. If the final position of a single sensor is known, the final positions of the other sensors are computed from the equalities $y_{i+1}-y_{i}=$ $r_{i}+r_{i+1}, \quad i=1,2, \ldots, n-1$. These equalities ensure that contiguous property is 
secured and no overlap is spared.

Remark When $R<L$, the contiguous 'MinSum' optimization problem does not use the fact whether the initial positions of sensors are located in the interval $[0, L]$ or not.

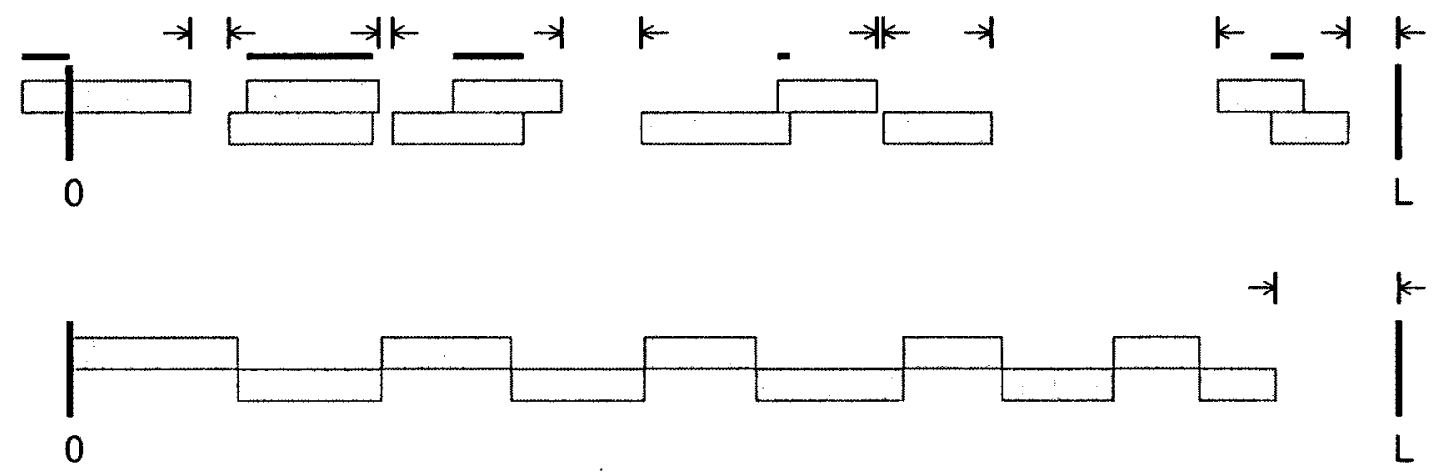

Figure 3.3: An illustration of contiguous coverage $(R<L)$ for the line segment $[0, L]$ employing 'MinSum' LP technique with nonidentical sensing range. The number of sensors is 10. The top and bottom line segments show the initial and final positions of the sensors with their sensing rages.

Figure 3.3 depicts an instance of contiguous coverage. There is only one gap due to $R<L$ and contiguity constraints. Similar to noncontiguous case, the coverage improves $28.05 \%$ for this example and this is the maximal possible coverage.

\subsubsection{Linearized 'MinSum' LP formulation}

Note that although the constraints of the 'MinSum' LP formulations presented in Sec. 3.2 are linear, the objective function is nonlinear. To linearize the objective function, we introduce new variables $z_{i}, i=1,2, \ldots, n$. We replace the objective functions in 
Eq. 3.2 with the following:

$$
\operatorname{minimize} \sum_{i=1}^{n} z_{i}
$$

We also add constraints in Eqs. 3.14, and 3.15 to all the LP formulations presented in Sec. 3.2 .

$$
\begin{aligned}
& z_{i} \geq x_{i}-y_{i}, \quad i=1,2, \ldots, n \\
& z_{i} \geq-\left(x_{i}-y_{i}\right), \quad i=1,2, \ldots, n
\end{aligned}
$$

To learn more on how to linearize LP objective function, see the work in [52].

\subsubsection{When initial positions are not necessarily inside $[0, L]$}

If the initial positions are not inside the interval $[0, L]$, no changes require for $R<L$. As in this case all the sensors have to move inside the line segment to maximize the coverage. However, for $R>L$, we need to reformulation the LP as the following:

$$
\begin{aligned}
\text { minimize } & \sum_{i=a}^{b}\left|x_{i}-y_{i}\right| \\
\text { subject to: } \quad & y_{a} \leq r_{a} \\
& y_{i+1}-y_{i} \leq r_{i}+r_{i+1}, \quad i=a, a+1, a+2, \ldots, b-1 \\
& y_{b} \geq L-r_{b}
\end{aligned}
$$

Here $S_{a}, S_{b}$ are pair of sensors such that the condition $1<a<b<n$ holds. The linearized scheme for the objective function remains the same as described in Sec. 3.2 .4 


\section{3 'MinMax' linear programming formulation}

The 'MinSum' LP formulations, as described in Sec. 3.2, minimize the sum of distances traversed by all sensors. In this method, few sensors may have to move a larger length than that of other sensors. Instead of minimizing the sum of distances traversed by all sensors, we may minimize the maximum distance by a sensor among all the sensors. We refer to this alternative approach as 'MinMax' LP method. In 'MinMax' LP formulations, only the objective function changes, all the constraints remains the same for both cases $(R>L$ and $R<L)$. The objective function in 'MinMax' LP formulation:

$$
\operatorname{minimize} \max _{1 \leq i \leq n}\left|x_{i}-y_{i}\right|
$$

To linearize the objective function in Eq. 3.20, we introduce a single variable $\alpha$. The objective function in Eq. 3.20 is replaced with the following:

$$
\text { minimize } \alpha
$$

We also add the following constraints:

$$
\begin{aligned}
& \alpha \geq x_{i}-y_{i}, \quad i=1,2, \ldots, n \\
& \alpha \geq-\left(x_{i}-y_{i}\right), \quad i=1,2, \ldots, n
\end{aligned}
$$




\subsection{Simulation}

We conducted extensive simulations to understand the impact of different coverage approaches. We developed a program using $\mathrm{C}++$ that incorporates the IBM ILOG Optimizer [53] to solve the LP formulation. We applied the optimization framework by varying the number of sensors planted randomly on a line segment. For each set up, we repeated the process 25 times. As the results have a similar trend, we show one result for each set up. We ran all simulations on a computer with $2 \mathrm{GHz}$ central processing unit speed and $3 \mathrm{~GB}$ random access memory. In case of complete coverage $(R>L)$, we take $R=1.1 L$, whereas when the a complete coverage is not possible $(R<L)$, we take $R=0.9 L$.

\subsubsection{Time complexity of LP and algorithmic approach}

To compare the LP technique and algorithmic approach in terms of time complexity, we vary the number of sensors planted each time. Then we applied LP and algorithmic technique proposed in MinMax [21] and MinSum [22] at least 25 times. We checked the trend of the simulation results and confirmed the similarity. Tables 3.1, 3.2 , and 3.3 list the simulation results on time complexity of LP and algorithmic techniques with complete, noncontiguous and contiguous coverage respectively. Figures $3.4,3.5$, and 3.6 show visual presentations of results on time complexity of LP and algorithmic techniques with complete, noncontiguous and contiguous coverage respectively. The time complexity of LP techniques is little less than that of the algorithmic approaches. For example when $n=100$, the 'MinSum' LP technique requires 0.18 second and 'MinSum' algorithmic approach requires 0.22 second to achieve a complete 
coverage. With same number of sensors, the 'MinMax' LP technique requires 0.20 second and 'MinMax' algorithmic approach requires 0.23 second to achieve a complete coverage. Again when $n=100$, the noncontiguous 'MinSum' LP technique requires 0.34 second and 'MinSum' algorithmic approach requires 0.38 second. With same number of sensors, the noncontiguous 'MinMax' LP technique requires 0.27 second and 'MinMax' algorithmic approach requires 0.31 second. With $n=100$, the contiguous 'MinSum' LP technique requires 0.19 second and 'MinSum' algorithmic approach requires 0.17 second. With same number of sensors, the contiguous 'MinMax' LP technique requires 0.16 second and 'MinMax' algorithmic approach requires 0.21 second. The little difference in time complexity between LP technique and algorithmic approach is due to efficiency of coding.

TABLE 3.1: A comparison of time complexity between LP and algorithmic approaches with complete coverage $(R>L)$. Both 'MinSum' and 'MinMax' versions of the objective functions are considered.

\begin{tabular}{r|rr|rr}
\hline & \multicolumn{2}{|c}{ MinSum } & \multicolumn{2}{c}{ MinMax } \\
No. of sensors $(n)$ & LP Framework & Algorithmic & LP Framework & Algorithmic \\
\hline 10 & 0.02 & 0.14 & 0.02 & 0.14 \\
20 & 0.14 & 0.16 & 0.15 & 0.16 \\
50 & 0.16 & 0.18 & 0.18 & 0.19 \\
100 & 0.18 & 0.22 & 0.20 & 0.23 \\
200 & 0.22 & 0.36 & 0.25 & 0.23 \\
500 & 0.39 & 1.15 & 0.41 & 1.07 \\
1000 & 1.29 & 4.30 & 1.21 & 3.66 \\
\hline
\end{tabular}

\subsubsection{The impact of contiguity requirement}

When the sum of sensing range $(R)$ is smaller than the line segment $(L)$, it is not possible to provide a complete coverage. However the coverage can be improved by 
TABLE 3.2: A comparison of time complexity between LP and algorithmic approaches with noncontiguous maximal coverage (when $R<L$ and more than one gaps are possible). Both 'MinSum' and 'MinMax' versions of the objective functions are considered.

\begin{tabular}{r|rr|rr}
\hline & \multicolumn{2}{|c|}{ MinSum } & \multicolumn{2}{c}{ MinMax } \\
No. of sensors $(n)$ & LP Framework & Algorithmic & LP Framework & Algorithmic \\
\hline 10 & 0.15 & 0.16 & 0.02 & 0.14 \\
20 & 0.20 & 0.20 & 0.17 & 0.19 \\
50 & 0.24 & 0.25 & 0.21 & 0.22 \\
100 & 0.34 & 0.38 & 0.27 & 0.31 \\
200 & 0.58 & 0.71 & 0.41 & 0.52 \\
500 & 1.79 & 2.46 & 0.81 & 1.47 \\
1000 & 6.97 & 9.44 & 2.94 & 5.68 \\
\hline
\end{tabular}

TABLE 3.3: A comparison of time complexity between LP and algorithmic approaches with contiguous maximal coverage (when $R<L$ ) and only one gap is possible. Both 'MinSum' and 'MinMax' versions of the objective functions are considered.

\begin{tabular}{r|rr|rr}
\hline & \multicolumn{2}{|c}{ MinSum } & \multicolumn{2}{c}{ MinMax } \\
No. of sensors $(n)$ & LP Framework & Algorithmic & LP Framework & Algorithmic \\
\hline 10 & 0.01 & 0.01 & 0.01 & 0.02 \\
20 & 0.03 & 0.04 & 0.04 & 0.05 \\
50 & 0.08 & 0.07 & 0.11 & 0.09 \\
100 & 0.19 & 0.17 & 0.16 & 0.21 \\
200 & 0.35 & 0.34 & 0.38 & 0.41 \\
500 & 0.62 & 0.65 & 0.68 & 0.71 \\
1000 & 1.13 & 1.24 & 1.34 & 1.19 \\
\hline
\end{tabular}

utilizing the overlaps to fill the gaps. To find the impact of contiguity requirement, we ran the simulation with different number of sensors. Each time the sensors are planted randomly. Then we apply the 'MinSum' and 'MinMax' LP techniques for both noncontiguous and contiguous requirements. We calculate the sum of distances traversed by all sensors and the improvement of coverage. We repeated each simulation setting at least 25 times and confirmed that the trend were similar. Tables 


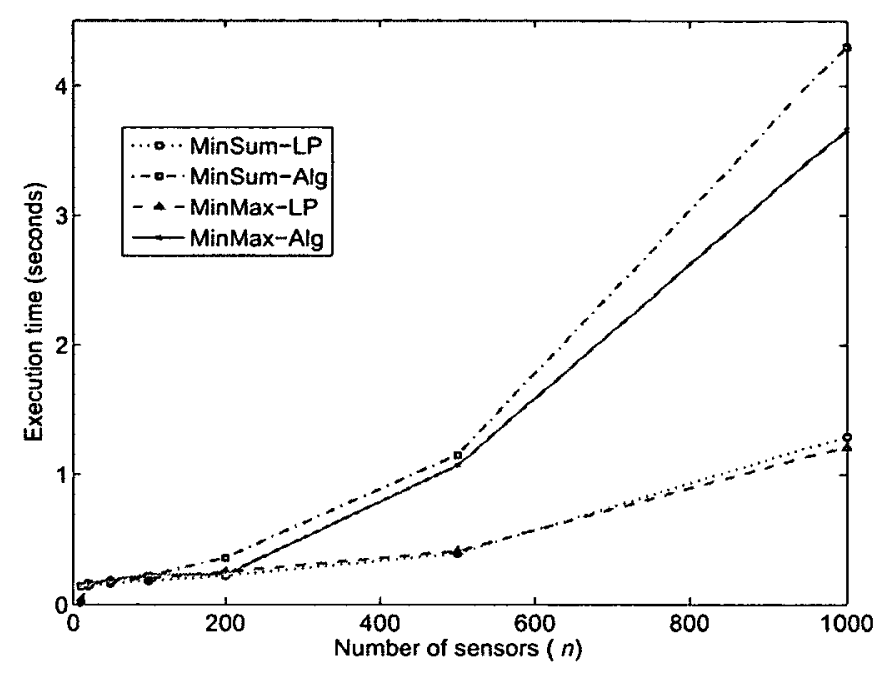

FIGURE 3.4: A comparison between LP and algorithmic approaches in terms of time complexity with complete coverage $(R>L)$.

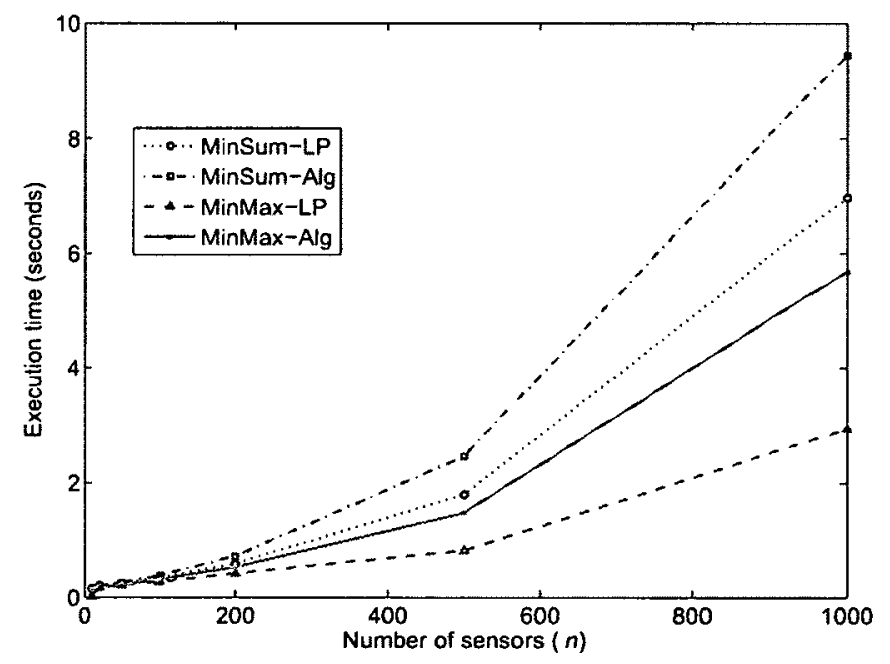

FIGURE 3.5: A comparison between LP and algorithmic approaches in terms of time complexity with noncontiguous maximal coverage (when $R<L$ and more than one gaps are possible).

3.4 and 3.5 compared noncontiguous and contiguous maximal coverage in terms of sum of distances traversed by all sensors for 'MinSum' and 'MinMax' LP techniques respectively. 


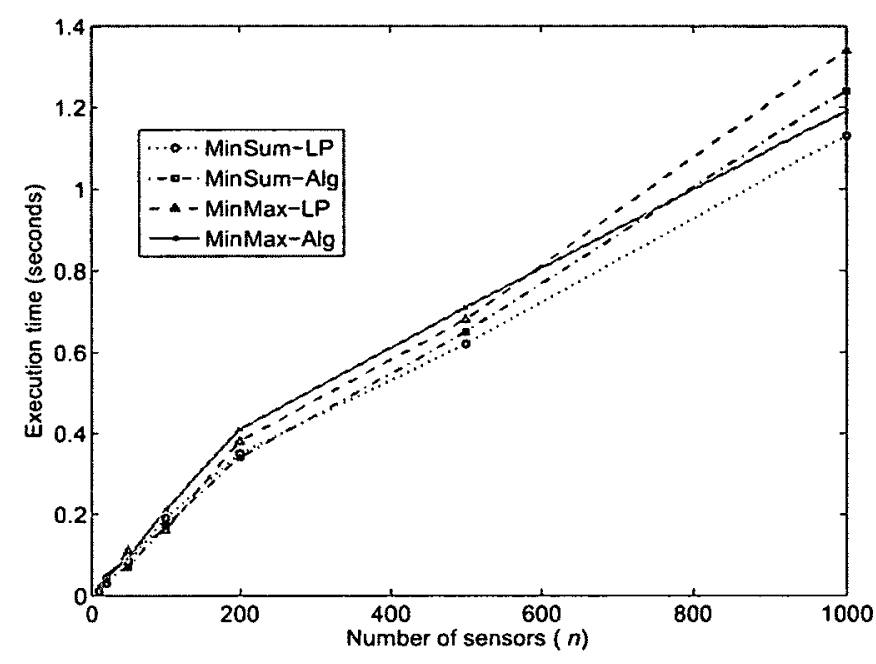

FIGURE 3.6: A comparison between LP and algorithmic approaches in terms of time complexity with noncontiguous maximal coverage (when $R<L$ and only one gap is possible).

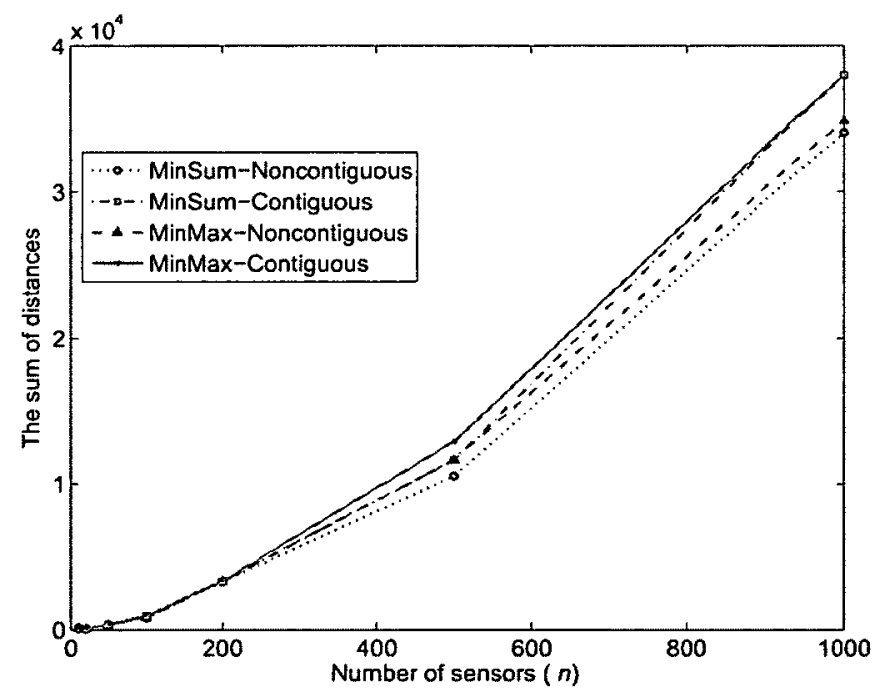

FiguRE 3.7: The difference between noncontiguous vs contiguous LP techniques in terms of sum distance traversed.

One important findings is that for both noncontiguous and contiguous case, the 
TABLE 3.4: The difference between noncontiguous vs contiguous 'MinSum' LP techniques in terms of sum distance traversed.

\begin{tabular}{r|rr|r|r}
\hline No. of sensors $(n)$ & Noncontiguous & Contiguous & $\begin{array}{r}\text { Extra distance } \\
\text { for Contiguity }\end{array}$ & $\begin{array}{r}\text { Coverage } \\
\text { Improved }\end{array}$ \\
\hline 10 & 72.70 & 84.50 & $16.23 \%$ & $31.81 \%$ \\
20 & 53.00 & 75.00 & $41.51 \%$ & $24.15 \%$ \\
50 & 317.40 & 339.90 & $7.09 \%$ & $33.70 \%$ \\
100 & 769.90 & 911.90 & $18.44 \%$ & $33.49 \%$ \\
200 & 3252.70 & 3300.90 & $1.48 \%$ & $34.37 \%$ \\
500 & 10527.10 & 11652.30 & $10.69 \%$ & $34.25 \%$ \\
1000 & 33992.30 & 37999.70 & $11.79 \%$ & $33.78 \%$ \\
\hline
\end{tabular}

TABLE 3.5: The difference between noncontiguous vs contiguous 'MinMax' LP techniques in terms of sum distance traversed.

\begin{tabular}{c|rr|r|r}
\hline No. of sensors $(n)$ & Noncontiguous & Contiguous & $\begin{array}{c}\text { Extra distance } \\
\text { for Contiguity }\end{array}$ & $\begin{array}{c}\text { Coverage } \\
\text { Improved }\end{array}$ \\
\hline 10 & 75.70 & 84.50 & $11.62 \%$ & $31.81 \%$ \\
20 & 67.00 & 75.20 & $12.24 \%$ & $24.15 \%$ \\
50 & 329.90 & 339.90 & $3.03 \%$ & $33.70 \%$ \\
100 & 819.90 & 919.10 & $12.10 \%$ & $33.49 \%$ \\
200 & 3285.10 & 3300.90 & $0.48 \%$ & $34.37 \%$ \\
500 & 11652.30 & 12946.00 & $9.99 \%$ & $34.25 \%$ \\
1000 & 34832.90 & 37999.70 & $9.09 \%$ & $33.78 \%$ \\
\hline
\end{tabular}

improvement of the coverage is always equal no matter which LP techniques ('MinSum' or 'MinMax') are applied. This is because the sum of the sensing range is equal for both contiguous and noncontiguous cases with different arrangement. For example, with $n=100$ the improvement of coverage is $33.49 \%$. Also in all cases, the improvements of coverage show the efficacy of the LP techniques. The sum of distances traversed by all sensors is higher in contiguous case. For example when $n=100$, the sum of distances traversed is $18.44 \%$ higher for contiguous 'MinSum' LP technique compared to the noncontiguous 'MinSum' LP technique. Also when 
$n=100$, the sum of distances traversed is $12.10 \%$ higher for contiguous 'MinMax' LP technique compared to the noncontiguous 'MinMax' LP technique. Figure 3.7 shows the difference between noncontiguous vs contiguous LP techniques in terms of sum distance traversed for both 'MinSum' and 'MinMax' objectives. For all methods, the sum of distances traversed increases with increased number of sensors. Also the sum of distances traversed by all sensors is higher in contiguous case. 


\section{Chapter 4}

\section{Line Segment Barrier Coverage with Di- minishing Sensing Range}

Sensors move to optimal positions from their initial positions to provide a better coverage. In chapter 3 , we provided an optimization framework that achieves such a goal with minimal movement to reduce energy consumption due to movement. At optimal relocations, we considered that sensors are still able to sense with the same range. We mentioned before that sensors have limited battery life and it is not feasible to replace batteries on numerous of sensors needed in most of the applications. As sensors consume energy whenever they move, the sensing range could be affected and may not be the same as before after relocation. In this chapter, we consider that sensing ranges decrease when sensors move. We provide an optimization framework to find the optimal relocations such that barrier coverage improves with minimal distance traversed by the sensors.

\subsection{Modified network model}

Just like the previous model in Sec. 3.1, there are $n$ sensors planted randomly to cover a closed line interval $I=[0, L]$. They are sorted by coordinate. The initial positions of sensors are $x_{1}, x_{2}, \ldots, x_{n}$ which may be either the inside or the outside of the line $I$, and the corresponding final positions are $y_{1}, y_{2}, \ldots, y_{n}$. At the beginning 
when sensors have full battery charge, the sensing range of the $i$-th sensor is $r_{i}$, where $1 \leq i \leq n$. Each time a sensor moves, its sensing range diminishes. As each sensor moves a different distance to reach its final position, it consumes different amount of energy according to its distance traveled. The work in [54] showed that the energy consumed by a sensor is linearly proportional to distance travelled. We assume that the amount of reduction of the sensing range is directly proportional to the amount of the distance travelled $\left(\Delta r_{i} \propto\left|x_{i}-y_{i}\right|\right)$. Therefore, the sensing range of the $i$-th sensor at optimal location is $r_{i}-\tau\left|x_{i}-y_{i}\right|$. Here $\tau$ is a proportional constant. So the $i$-th sensor at its final position covers an interval of length $2\left(r_{i}-\tau\left|x_{i}-y_{i}\right|\right)$. All the points inside the interval are considered as covered within the range of the sensor. Our focus is to improve the coverage with minimal movement of the sensors taken into the account that sensor ranges diminish as they move.

\subsection{Variable range 'MinSum' linear programming formulations}

Here we formulate the problem of forming a line segment barrier from initial random deployment of sensors as LP. However sensing ranges shorten as they move. When $R>L$, a complete coverage may be possible depending on the parameter $\tau$, and the amount of movements of the sensors. The LP solves for the optimal final positions of sensors such that sum of distances traversed by all sensors is minimized. When a complete coverage is not possible $(R<L)$, the LP formulation maximizes the coverage in two different arrangements: contiguous and noncontiguous. A contiguous 
arrangement of sensors attains the largest possible coverage $R$, if the provided coverage is a contiguous subinterval of $[0, L]$. A noncontiguous arrangement of sensors achieves the largest possible coverage $R$, when the provided coverage is a collection of disjoint subintervals. We present LP formulations which are valid when the initial positions of the sensors are inside the interval $[0, L]$.

\subsubsection{Variable range complete coverage 'MinSum' LP formu- lation $(R>L)$}

When $R>L$, a complete coverage is possible depending on the parameter $\tau$. The following constraints in Eqs. 4.2, 4.3, and 4.4 along with the objective function in Eq. 4.1 constitute the LP formulation:

$$
\begin{array}{cl}
\operatorname{minimize} & \sum_{i=1}^{n}\left|x_{i}-y_{i}\right| \\
\text { subject to: } & y_{1} \leq r_{1}-\tau\left|x_{1}-y_{1}\right| \\
& y_{i+1}-y_{i} \leq r_{i}-\tau\left|x_{i}-y_{i}\right|+r_{i+1}-\tau\left|x_{i+1}-y_{i+1}\right|, \\
& i=1,2, \ldots, n-1 \\
& y_{n} \geq L-\left(r_{n}-\tau\left|x_{n}-y_{n}\right|\right)
\end{array}
$$

The objective function in Eq. 4.1 minimizes the sum of distances traversed by all sensors. The constraints in Eq. 4.2, 4.3, and 4.4 ensure the complete coverage of the line segment.

Figure 4.1 depicts how sensors provide a complete coverage by moving to the final positions. There is no gap between sensors. The final positions are optimized employing LP technique such that the sum of distances traversed by all sensors are 


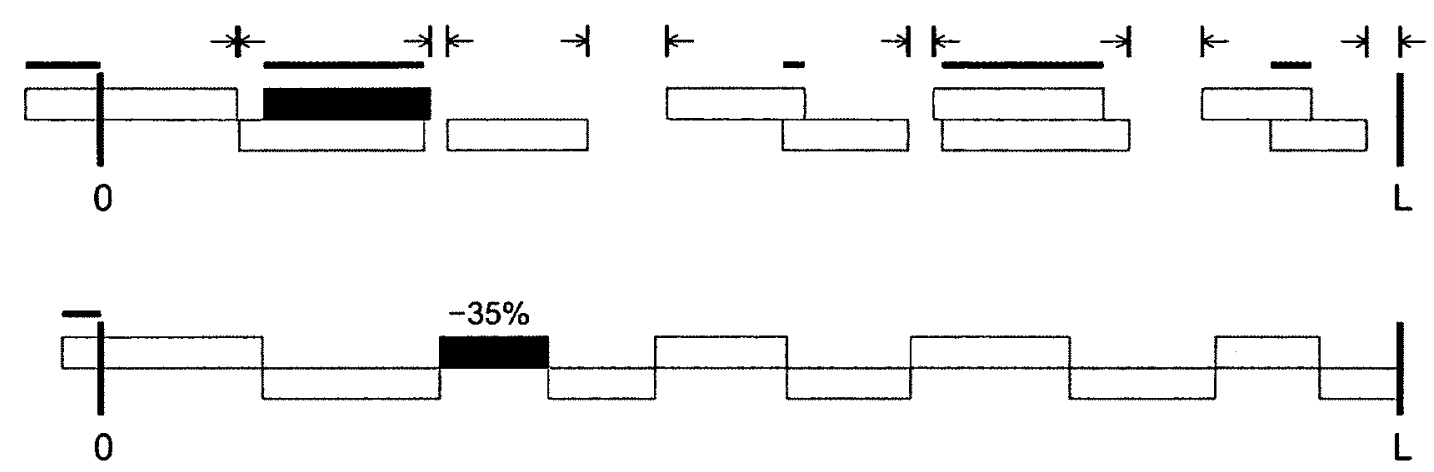

Figure 4.1: An instance of complete coverage $(R>L)$ for the line segment $[0, L]$ employing 'MinSum' LP technique with distant depended sensing range. The number of sensors is 10 . The top and bottom line segments show the initial and final positions of the sensors with their sensing rages.

minimized. For this particular example showed in the above figure 4.1, the sensor that moves largest distance (shown darker in the Fig. 4.1) among all sensors has shrunk its sensing range $35.13 \%$. The proportional constant $\tau=0.2$. The shrinkage of total sensing range of all sensors is $12.67 \%$. The coverage still improves $21.28 \%$. The complete coverage is possible as $\sum_{i}^{n}\left(r_{i}-\tau\left|x_{i}-y_{i}\right|\right)>L$. This formulation is a more generalized form of the LP model presented in Sec. 3.2.2.

\subsubsection{Variable range maximal coverage 'MinSum' LP formu- lations $(R<L)$}

When $R<L$, obviously a complete coverage is not possible. Just like the static sensing range, we will investigate two variants of LP formulations: noncontiguous and contiguous. 


\section{Variable range noncontiguous 'MinSum' LP}

With $R<L$ if the coverage is noncontiguous, there could exist more than a single gap. The Eqs. 4.5, 4.6, 4.7, and 4.8 constitute the LP formulation for noncontiguous arrangement with $R<L$.

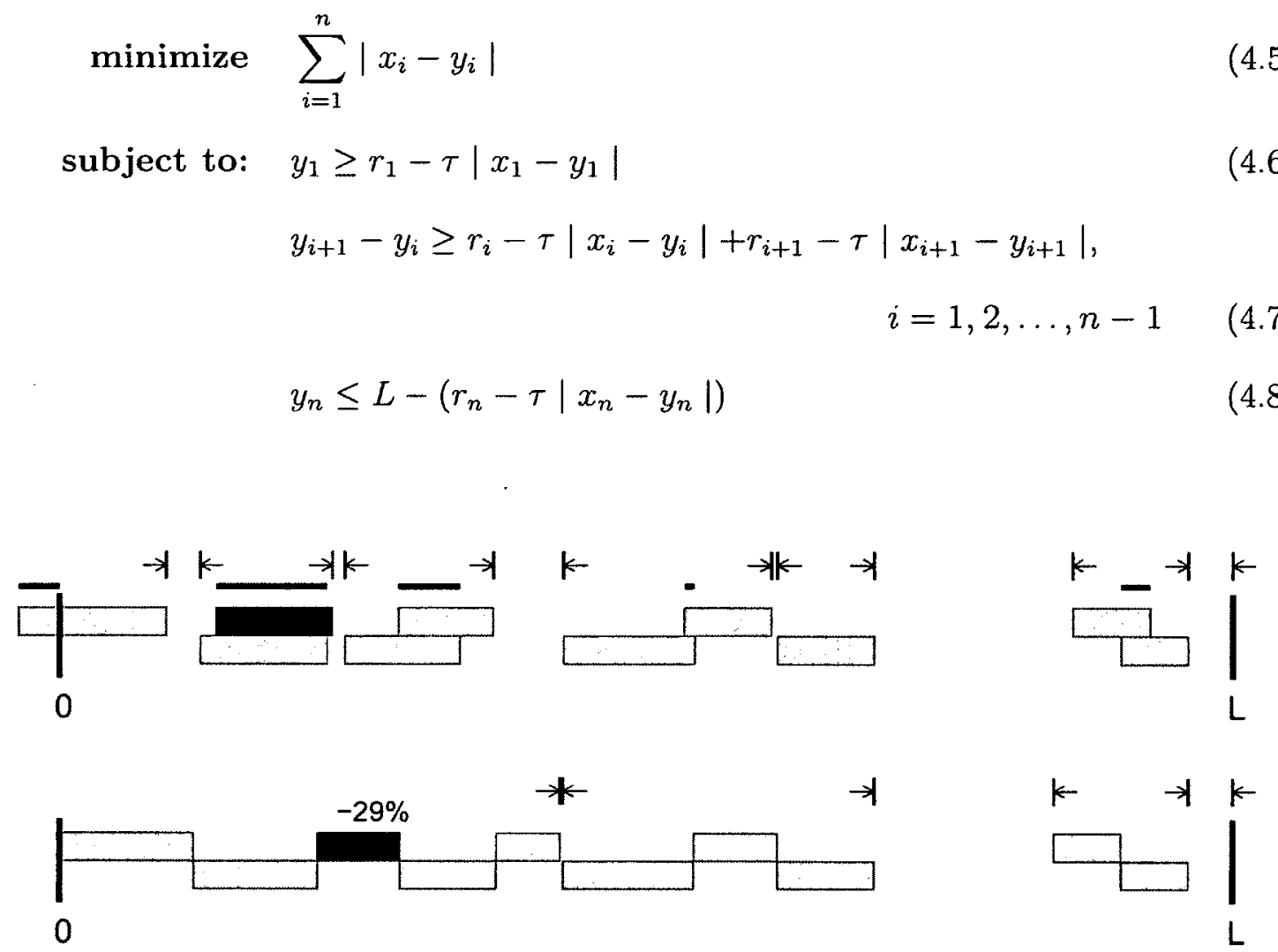

FIGURE 4.2: An instance of noncontiguous coverage $(R<L)$ for the line segment $[0, L]$ employing 'MinSum' LP technique with variable sensing range. The number of sensors is 10. The top and bottom line segments show the initial and final positions of the sensors with their sensing rages.

Figure 4.2 illustrates an example of noncontiguous coverage. Obviously there are multiple gaps due to $\sum_{i}^{n}\left(r_{i}-\tau\left|x_{i}-y_{i}\right|\right)<L$ and noncontiguous arrangement of the sensors. The proportional constant $\tau=0.2$. For this particular example showed 
in the above figure 4.2 , the shrinkage of total sensing range of all sensors is $10.91 \%$. The sensor that moves largest distance (shown darker in the Fig. 4.2) among all sensors has shrunk its sensing range $29.01 \%$. However, the coverage improves $17.43 \%$ when compared to the initial coverage. For $\tau=0$, this formulation converts to static sensing range LP model presented in Sec. 3.2.3.

\section{Variable range contiguous 'MinSum' LP}

A contiguous coverage requires that no overlap or gap exist between two adjacent sensors. With $R<L$ and distant dependent sensing range, the inequalities in Eq. 4.3 are replaced by the equalities in Eq. 4.11. The objective function in Eq. 4.1 changes as well. The Eqs. in 4.9, 4.10, and 4.12 define the LP formulation for continuous arrangement with $R<L$.

$$
\begin{array}{cl}
\text { minimize } & \left|x_{1}-y_{1}\right|+\left|x_{2}-\left(y_{1}+r_{1}+r_{2}\right)\right| \\
& +\sum_{i=3}^{n}\left|x_{i}-\left(y_{1}+r_{1}+r_{i}+2 \sum_{j=2}^{i-1} r_{j}\right)\right| \\
\text { subject to: } & y_{1} \geq r_{1}-\tau\left|x_{1}-y_{1}\right| \\
& y_{i+1}-y_{i}=r_{i}-\tau\left|x_{i}-y_{i}\right|+r_{i+1}-\tau\left|x_{i+1}-y_{i+1}\right|, \\
& \\
& y_{n} \leq L-\left(r_{n}-\tau\left|x_{n}-y_{n}\right|\right)
\end{array}
$$

The constraints in Eq. 4.10, and 4.12 ensure that entire sensing ranges of the endmost sensors of both sides are utilized to cover the part of the line segment. The constraints in Eq. 4.11 ensure that no overlap or gap exist between two adjacent sensors. Figure 4.3 depicts an example of contiguous coverage. There is only one gap due to contiguity 

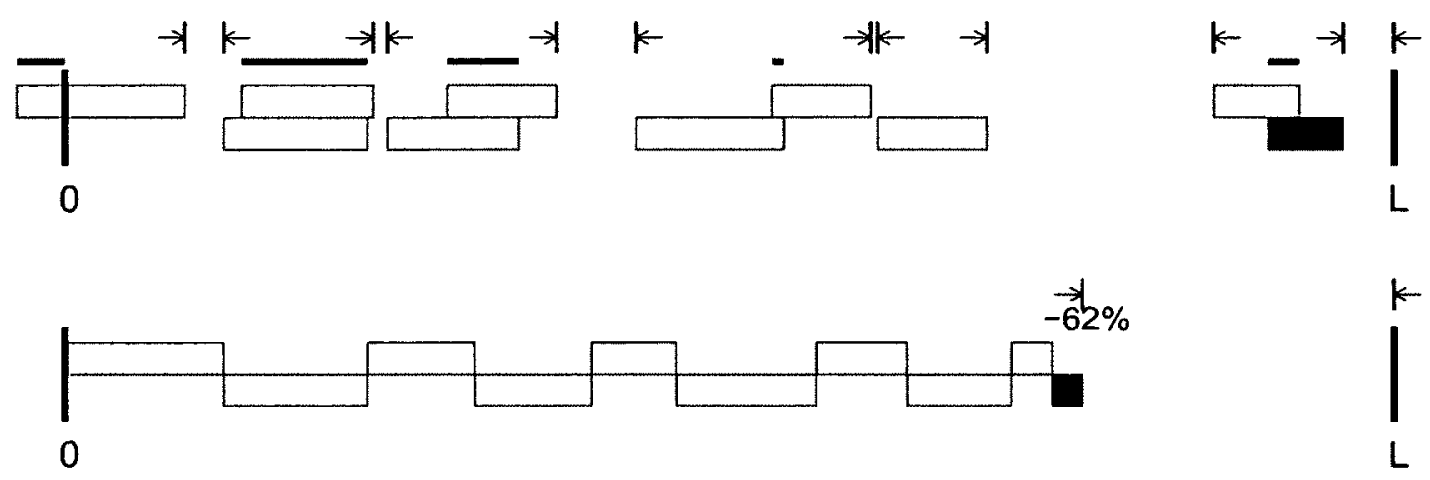

FiguRE 4.3: An illustration of contiguous coverage $(R<L)$ for the line segment $[0, L]$ employing 'MinSum' LP technique with variable sensing range. The number of sensors is 10. The top and bottom line segments show the initial and final positions of the sensors with their sensing rages.

constraints. Even with the proportional constant $\tau=0.1$, the coverage only improves $11.53 \%$ and this is the maximal possible coverage for the example of the above figure 4.3. The sensor that moves largest distance (shown darker in the Fig. 4.3) among all sensors has shrunk its sensing range $61.61 \%$. The shrinkage of total sensing range of all sensors is $15.39 \%$. This formulation is a more generalized form of the LP model presented in Sec. 3.2.3.

\subsubsection{Linearized 'MinSum' LP formulation}

Unlike the static sensing range case presented in Chapter 3, the variable range 'MinSum' LP framework in Sec. 4.2 contains nonlinear objective functions and constraints. The linearize procedures are similar for complete, contiguous and noncontiguous coverage cases. Here we show how to linearize the objective function and constraints only for the complete coverage case. We introduce new variables $z_{i}, i=1,2, \ldots, n$. The following formulation constitutes the variable range complete coverage 'MinSum' 
LP framework:

$$
\begin{aligned}
\text { minimize } & \sum_{i=1}^{n} z_{i} \\
\text { subject to: } \quad & y_{1} \leq r_{1}-\tau z_{1} \\
& y_{i+1}-y_{i} \leq r_{i}-\tau z_{i}+r_{i+1}-\tau z_{i+1}, \quad i=1,2, \ldots, n-1 \\
& y_{n} \geq L-\left(r_{n}-\tau z_{n}\right) \\
& z_{i} \geq x_{i}-y_{i}, \quad i=1,2, \ldots, n \\
& z_{i} \geq-\left(x_{i}-y_{i}\right), \quad i=1,2, \ldots, n
\end{aligned}
$$

We add constraints in Eqs. 4.17, and 4.18 because of the new variable $z_{i}$.

\subsection{Variable range 'MinMax' linear programming formulation}

The variable range 'MinSum' LP formulation, described in Sec. 4.2, minimizes the sum of distances traversed by all sensors. In this method, few sensors may have to move a larger length than that of other sensors. Instead of minimizing the sum of distances traversed by all sensors, we may minimize the maximum distance among all the sensors. We refer to this alternative approach as variable range 'MinMax' LP method. In 'MinMax' LP formulations, only the objective function changes, all the constraints remains the same for both cases $(R>L$ and $R<L)$. The objective 
function in 'MinMax' LP formulation:

$$
\operatorname{minimize} \max _{1 \leq i \leq n}\left|x_{i}-y_{i}\right|
$$

To linearize the objective function in Eq. 4.19, we introduce a single variable $\alpha$. The objective function in Eq. 4.19 is replaced with the following:

$$
\text { minimize } \alpha
$$

However we still have nonlinear constraints. We replace $\left|x_{i}-y_{i}\right|$ by $z_{i}$ and add the following constraints:

$$
\begin{aligned}
& \alpha \geq z_{i}, \quad i=1,2, \ldots, n \\
& \alpha \geq-z_{i}, \quad i=1,2, \ldots, n \\
& z_{i} \geq x_{i}-y_{i}, \quad i=1,2, \ldots, n \\
& z_{i} \geq-\left(x_{i}-y_{i}\right), \quad i=1,2, \ldots, n
\end{aligned}
$$

\subsection{Variable range 'MinSum' heuristic algorithm}

In this subsection, we propose a heuristic algorithm. When a complete coverage is possible, the heuristic algorithm provides a coverage close to a complete one with suboptimal relocations of the sensors. When a complete coverage is not possible, the heuristic algorithm provides noncontiguous maximal coverage with relocations of the sensors very close to suboptimal positions. In terms of coverage, the heuristic solution provides almost the same level of coverage to that of the LP techniques. The solution 
is suboptimal as the sum of distances traversed by all sensors is minimized as much as possible.

The heuristic algorithm starts removing the gaps from left to right. For each gap (starting with the leftmost), say $g$ we first search the 'closest overlap' on left. If there are sensors attached to each other between the gap and the overlap, the overlap should be large enough to fill any new gap emerging from the shrinkage of the sensing range. If the overlap is not large enough to fill the gap, the algorithm tries to fill the gap with the next 'closest overlap' on left. If there is no more overlap on the left, the algorithm fills the gap utilizing the 'closest overlap' from the right. There is possibility that the gap may not be filled. The overlaps on both sides could be far away, therefore moving sensors could create more gaps due to shrinkage of sensing range. The algorithm then tries to fill the next gap with the same procedure. The final positions may not be optimal, as gaps are not filled by comparing the cost from both sides. The algorithm avoids moving sensors in both direction, choosing closest overlap from one side, and (if the gap is not filled) choosing closest overlap from the other side produces results very close to optimal one. At any time a cheap overlap in terms of movement cost (if not the cheapest) is used to reduce the gap.

Definition 4.4.1 (Right shift) Let $g$ be a gap, $o_{j}$ be the closest overlap of sensors to the left of the gap $g$, and $x_{i}, x_{i-1}, \ldots, x_{j}$ are the sequence of sensors starting from the sensor left of gap $g$ until the right overlapping sensor. Let the size of $o_{j}$ be $\left|o_{j}\right|$. $c_{i}, c_{i-1}, \ldots, c_{j}$ are the amount of right shifts of the corresponding sensors. Let there be $p$ sensors between the gap and the overlap. Therefore $j=i-p+1$.

Definition 4.4.2 (Right shift cost) To fill the entire gap $g$, i-th sensor needs to move $c_{i}^{\text {req }}$ distance. The $i$-th sensor can move at most $c_{i}^{\text {max }}$ without creating a gap. 
If $c_{i}^{r e q} \leq c_{i}^{\text {max }}$, then $c_{i}=c_{i}^{r e q}$, else $c_{i}=c_{i}^{\text {max }}$. Once $c_{i}$ is computed, the other shifting amount can be found from the relation $c_{i-1}=\frac{1+\tau}{1-\tau} c_{i}$.

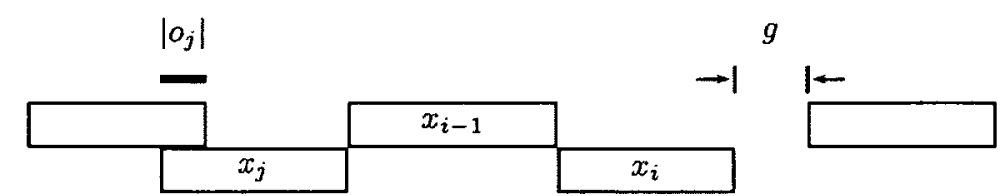

FIGURE 4.4: An instance of right shift. Here $p=3$, therefore $j=i-p+1=i-2$.

Two sensors are in attached position if there is no overlap or gap between them. If $j \neq i$, there is at least one pair of sensors in attached position. As the sensors move to the right to fill the gap, previous attached sensors still need to be in attached position. Consider a pair of sensors $i$ and $i-1$ in attached positions. See Fig. 4.4. Because of the movement the $i-1$-th sensor's right side sensing range moves from $x_{i-1}+r_{i-1}$ to $x_{i-1}+c_{i-1}+r_{i-1}-\tau c_{i-1}$. However, it has to cover $i$-th sensor right shift $\left(c_{i}\right)$ and the shrinkage amount $\left(\tau c_{i}\right)$. Therefore, sensors $i$ and $i-1$ will be in attached position after moving distances $c_{i}$ and $c_{i-1}$ if the following condition hold:

$$
\begin{aligned}
& x_{i-1}+c_{i-1}+r_{i-1}-\tau c_{i-1}-\left(x_{i-1}+r_{i-1}\right)=(1+\tau) c_{i} \\
& c_{i-1}-\tau c_{i-1}=(1+\tau) c_{i} \\
& c_{i-1}=\frac{1+\tau}{1-\tau} c_{i} \\
& c_{i-2}=\left[\frac{1+\tau}{1-\tau}\right]^{2} c_{i} \\
& c_{j}=\left[\frac{1+\tau}{1-\tau}\right]^{p-1} c_{i}
\end{aligned}
$$

Let $c_{j}^{\max }$ is the maximum of right shift available due to the size of the overlap $\left|o_{j}\right|$. 
For the $j$-th sensor, we have the following condition:

$$
\begin{aligned}
& x_{j}+c_{j}-\left(r_{j}-\tau c_{j}\right)-\left(x_{j}-r_{j}\right) \leq\left|o_{j}\right| \\
& c_{j}+\tau c_{j} \leq\left|o_{j}\right| \\
& c_{j}^{\max }=\frac{\left|o_{j}\right|}{1+\tau}
\end{aligned}
$$

From Eqs. 4.28 and 4.27

$$
c_{i}^{\max }=\left[\frac{1-\tau}{1+\tau}\right]^{p-1} \frac{\left|o_{j}\right|}{1+\tau}
$$

We want to find the amount of shift required to fill the entire size of the gap $|g|$.

$$
\begin{aligned}
& x_{i}+c_{i}^{r e q}+r_{i}-\tau c_{i}^{r e q}-\left(x_{i}+r_{i}\right)=|g| \\
& c_{i}^{r e q}-\tau c_{i}^{r e q}=|g| \\
& c_{i}^{r e q}=\frac{|g|}{1-\tau}
\end{aligned}
$$

Definition 4.4.3 (Left shift) Let $g$ be a gap, $o_{k}$ be the closest overlap of sensors to the right of the gap $g$, and $x_{i}, x_{i+1}, \ldots, x_{k}$ are the sequence of sensors starting from the sensor right of gap $g$ until the left overlapping sensor. Let the size of $o_{k}$ be $\left|o_{k}\right|$. $c_{i}, c_{i-1}, \ldots, c_{k}$ are the amount of left shifts of the corresponding sensors. Let there be $q$ sensors between the gap and the overlap. Therefore $k=i+q-1$.

Definition 4.4.4 (Left shift cost) To fill the entire gap $g$, i-th sensor needs to move $c_{i}^{r e q}$ distance. The $i$-th sensor can move at most $c_{i}^{\text {max }}$ without creating a gap. If $c_{i}^{\text {req }} \leq c_{i}^{\max }$, then $c_{i}=c_{i}^{r e q}$, else $c_{i}=c_{i}^{\max }$. Once $c_{i}$ is computed, the other shifting amount can be found from the relation $c_{i-1}=\frac{1+\tau}{1-\tau} c_{i}$. 


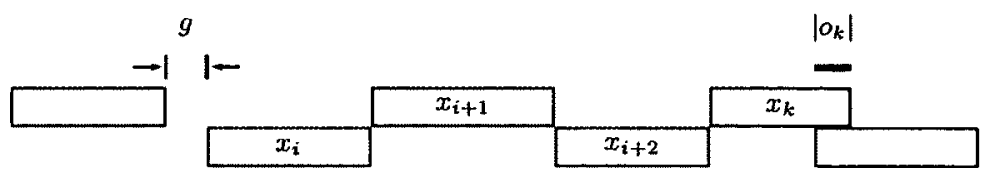

FiguRE 4.5: An instance of left shift. Here $q=4$, therefore $k=i+q-1=i+3$.

Following a similar procedure in Definitions 4.4 .1 and 4.4 .2 , we find the following results:

$$
\begin{aligned}
& c_{i+1}=\frac{1+\tau}{1-\tau} c_{i} \\
& c_{k}=\left[\frac{1+\tau}{1-\tau}\right]^{q-1} c_{i}
\end{aligned}
$$

Figure 4.5 illustrates an instance of left shift. Let $c_{k}^{\max }$ is the maximum of left shift available due to the size of the overlap $\left|o_{k}\right|$. For the $k$-th sensor we have

$$
c_{k}^{\max }=\frac{\left|o_{k}\right|}{1+\tau}
$$

From Eqs. 4.33 and 4.32

$$
c_{i}^{\max }=\left[\frac{1-\tau}{1+\tau}\right]^{q-1} \frac{\left|o_{k}\right|}{1+\tau}
$$

We want to find the amount of shift required to fill the entire size of the gap $|g|$.

$$
c_{i}^{r e q}=\frac{|g|}{1-\tau}
$$

Algorithm 1 provides a heuristic solution of the variable range 'MinSum' problem. 


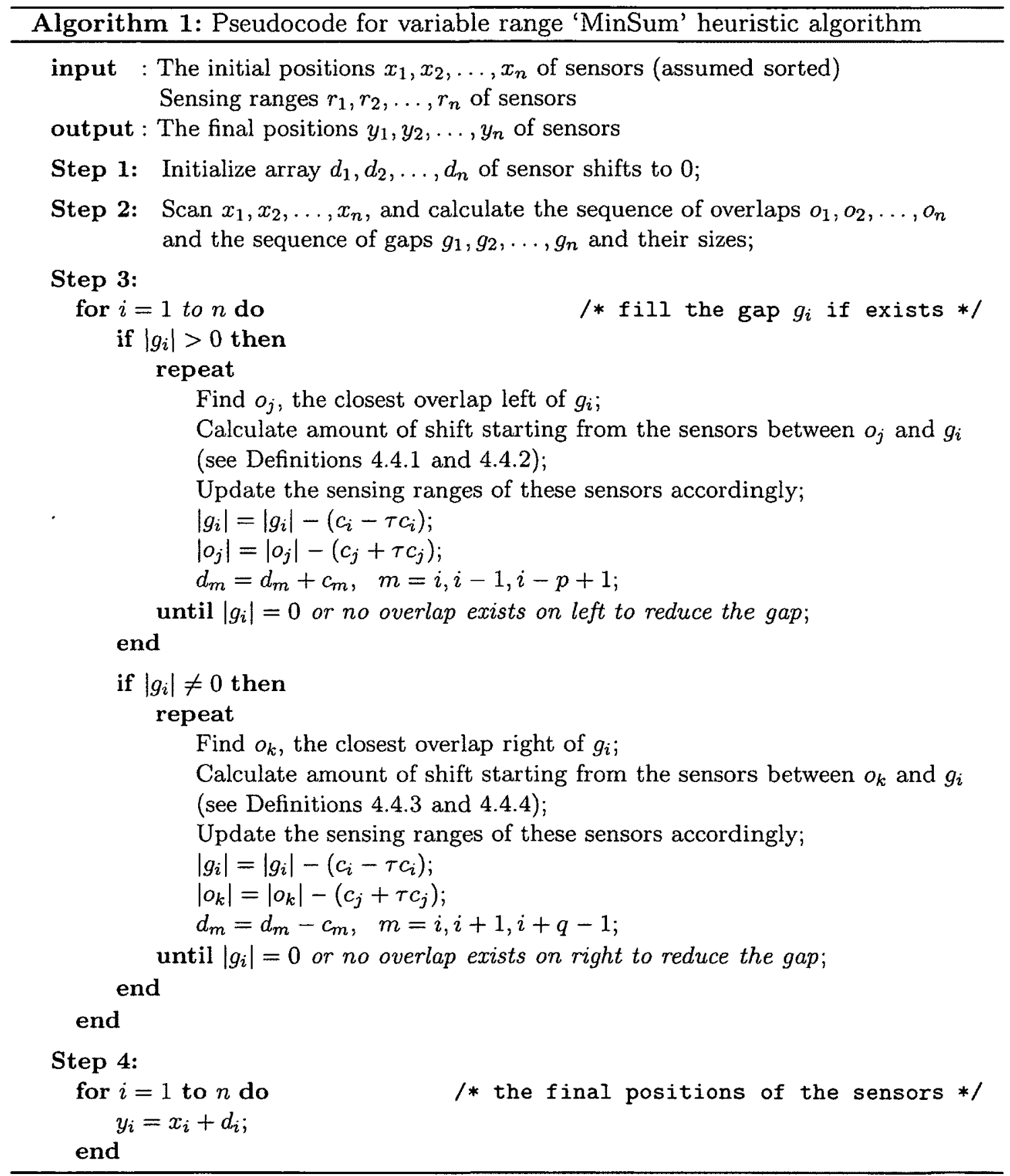




\subsection{Simulation}

In this section, we present the simulation results to understand the challenges imposed by the reduction of range due to mobility. We also present simulation results of the heuristic algorithm to prove its effectiveness. We used the IBM ILOG Optimizer [53] to solve the LP formulation. Similar to static range case, we applied the optimization framework by varying the number of sensors planted randomly on a line segment. For each set up, we repeated the process 25 times. As the results have a similar trend, we present one result for each set up. We ran all simulations on a computer with $2 \mathrm{GHz}$ central processing unit speed and 3 GB random access memory. In case of complete coverage $(R>L)$, we take $R=1.1 L$, whereas when the a complete coverage is not possible $(R<L)$, we take $R=0.9 L$.

\subsubsection{Time complexity of variable range LP technique}

TABLE 4.1: A comparison of execution time of different LP techniques to improve the coverage with minimal movement of sensors. Both 'MinSum' and 'MinMax' versions of the objective functions are considered. The proportional constant $\tau=0.1$

\begin{tabular}{r|rrrr|rr}
\hline & \multicolumn{2}{|c}{$\begin{array}{c}\text { Complete }(R>L) \\
\text { 'MinSum' }\end{array}$} & \multicolumn{2}{c}{ 'Moncontiguous $(R<L)$} & \multicolumn{2}{c}{ Contiguous $(R<L)$} \\
$n$ & 'MinMax' & 'MinSum' & 'MinMax' & 'MinSum' & 'MinMax' \\
\hline 10 & 0.02 & 0.14 & 0.02 & 0.15 & 0.02 & 0.02 \\
20 & 0.16 & 0.17 & 0.16 & 0.18 & Inf $^{\dagger}$ & Inf $^{\dagger}$ \\
50 & 0.18 & 0.20 & 0.2 & 0.25 & Inf $^{\dagger}$ & Inf $^{\dagger}$ \\
100 & 0.23 & 0.28 & 0.3 & 0.38 & Inf $^{\dagger}$ & Inf $^{\dagger}$ \\
200 & 0.34 & 0.48 & 0.5 & 0.76 & Inf $^{\dagger}$ & Inf $^{\dagger}$ \\
500 & 0.69 & 1.46 & 1.16 & 2.71 & Inf $^{\dagger}$ & Inf $^{\dagger}$ \\
1000 & 2.51 & 5.23 & 4.74 & 10.78 & Inf $^{\dagger}$ & Inf $^{\dagger}$ \\
\hline
\end{tabular}

†Infeasible

To compare the time complexity of different coverage approaches (complete or 
maximal), we vary the number of sensors planted each time. Then we applied LP technique at least 25 times. We checked the trend of the simulation results and confirmed the similarity. Table 4.1 lists the simulation results on execution time (in second) with complete, noncontiguous and contiguous coverage models. Figure 4.6 shows a visual presentation of the results in terms of time complexity with complete and noncontiguous respectively. The first important finding is that the variable range contiguous LP technique (both 'MinSum' and 'MinMax' approaches) is infeasible almost in all cases. The reason is that most of the sensors in this technique have to traverse a long way to ensure the contiguity. In doing so, their sensing ranges reduce significantly. This leads to violations of constraints and the LP solution appears infeasible.

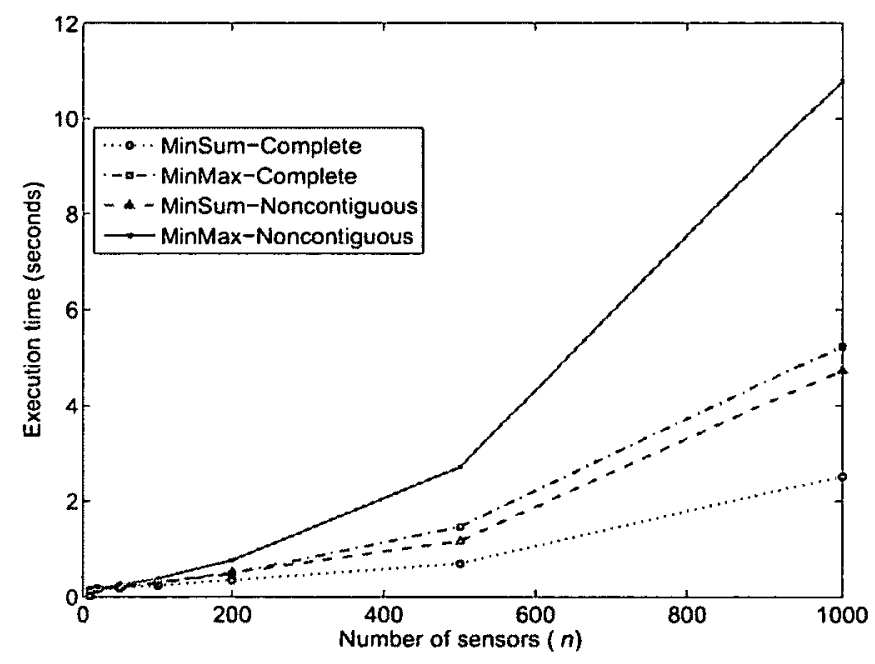

Figure 4.6: A comparison of execution time of different LP techniques. The proportional constant $\tau=0.1$

All variants of LP techniques require a fraction of seconds when the number of sensors is less than 1000. There is not significant difference in the execution time of complete coverage and noncontiguous coverage LP techniques. For example when 
$n=100$, the variable range complete coverage 'MinSum' LP technique requires 0.23 second, whereas the variable range noncontiguous 'MinSum' LP technique requires 0.30 second. With same number of sensors, the complete coverage 'MinMax' LP technique requires 0.28 second and noncontiguous 'MinMax' LP technique requires 0.38 second to solve the problem. Also 'MinMax' LP technique requires little longer time than 'MinSum' LP technique with few odd exceptions.

\subsubsection{Comparison between LP technique and heuristic method}

TABLE 4.2: A comparison between LP technique and heuristic algorithm in terms of coverage, execution time, and sum of distance traversed when $R>L$. The proportional constant $\tau=0.1$

\begin{tabular}{r|rrr|rr|r}
\hline & \multicolumn{3}{|c}{ Coverage } & \multicolumn{3}{c}{ Execution Time } \\
$n$ & Initial & LP & Heuristic & LP & Heuristic & Sum of distance \\
\hline 10 & $74.61 \%$ & $100.00 \%$ & $94.04 \%$ & 0.02 & 0.01 & $8.63 \%$ \\
20 & $80.87 \%$ & $100.00 \%$ & $93.65 \%$ & 0.16 & 0.03 & $9.91 \%$ \\
50 & $80.35 \%$ & $100.00 \%$ & $94.45 \%$ & 0.18 & 0.06 & $10.77 \%$ \\
100 & $77.37 \%$ & $100.00 \%$ & $98.25 \%$ & 0.23 & 0.10 & $11.26 \%$ \\
200 & $78.25 \%$ & $100.00 \%$ & $97.79 \%$ & 0.34 & 0.18 & $12.71 \%$ \\
500 & $77.56 \%$ & $100.00 \%$ & $93.93 \%$ & 0.69 & 0.31 & $13.96 \%$ \\
1000 & $77.39 \%$ & $100.00 \%$ & $96.21 \%$ & 2.51 & 0.67 & $9.66 \%$ \\
\hline
\end{tabular}

We ran the simulation with different number of sensors to show the effectiveness of the variable range LP technique and the proposed heuristic algorithm. Each time the sensors are planted randomly. For each set, we measured the initial and final coverage, execution time, and the difference of sum distanced traversed by all sensors. We repeated each simulation setting at least 25 times and confirmed that the trend were similar. Tables 4.2 and 4.3 depict a comparison between LP technique and the 
TABLE 4.3: A comparison between LP technique and heuristic algorithm in terms of coverage, execution time, and sum of distance traversed when $R<L$. The proportional constant $\tau=0.1$

\begin{tabular}{|c|c|c|c|c|c|c|}
\hline \multirow[b]{2}{*}{$n$} & \multicolumn{3}{|c|}{ Coverage } & \multicolumn{2}{|c|}{ Execution Time } & \multirow{2}{*}{$\begin{array}{r}\text { Gap } \\
\text { Sum of distance }\end{array}$} \\
\hline & Initial & LP & Heuristic & LP & Heuristic & \\
\hline 10 & $68.85 \%$ & $85.82 \%$ & $74.63 \%$ & 0.02 & 0.01 & $5.44 \%$ \\
\hline 20 & $76.78 \%$ & $93.17 \%$ & $81.34 \%$ & 0.16 & 0.04 & $8.26 \%$ \\
\hline 50 & $73.36 \%$ & $94.88 \%$ & $79.33 \%$ & 0.2 & 0.09 & $9.27 \%$ \\
\hline 100 & $74.17 \%$ & $94.30 \%$ & $87.14 \%$ & 0.3 & 0.17 & $6.75 \%$ \\
\hline 200 & $74.05 \%$ & $94.73 \%$ & $87.94 \%$ & 0.5 & 0.25 & $5.79 \%$ \\
\hline 500 & $74.34 \%$ & $95.23 \%$ & $88.10 \%$ & 1.16 & 0.43 & $8.58 \%$ \\
\hline 1000 & $74.68 \%$ & $94.79 \%$ & $86.94 \%$ & 4.74 & 0.78 & $9.11 \%$ \\
\hline
\end{tabular}

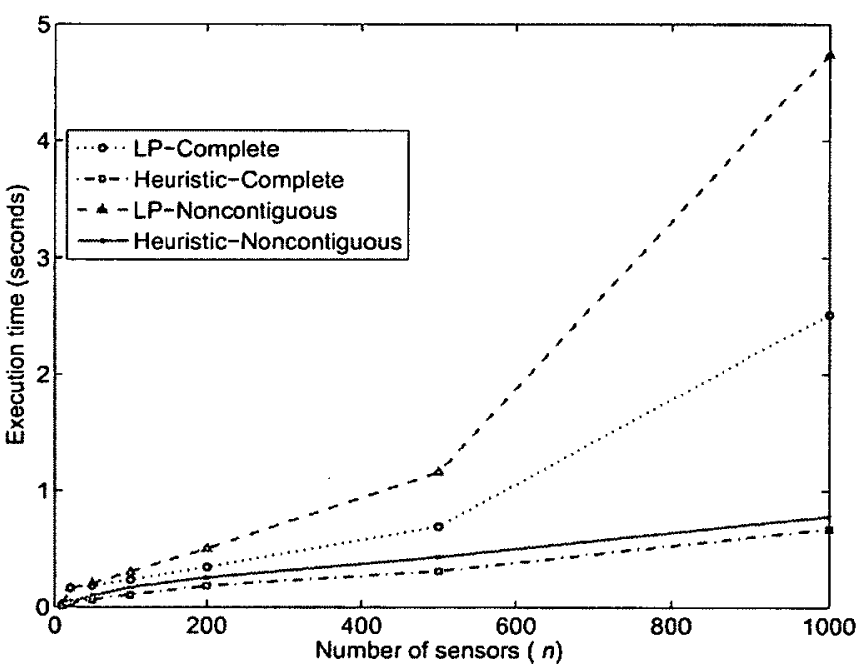

FIgURE 4.7: A comparison between LP technique and heuristic algorithm in terms of time. The proportional constant $\tau=0.1$

heuristic algorithm in terms of coverage, execution time, and sum of distances traversed when $R>L$ and $R<L$ respectively. Figure 4.7 compares the execution time (in second) between LP technique and heuristic algorithm in terms of time. When a complete coverage is possible $(R>L)$ the heuristic algorithm provides coverage close to $100 \%$ with better execution time when compared to LP technique. For example 
with $n=100$, the LP techniques provides $100 \%$ coverage, and the heuristic algorithm achieves $98.25 \%$ coverage. The LP technique requires 0.23 second, and the heuristic algorithm requires 0.10 second to solve the problem. In the heuristic algorithm, the sum of distances traversed by all sensors is $11.26 \%$ higher than that of the LP technique.

When a complete coverage is possible $(R<L)$, the heuristic algorithm provides coverage almost close to that of LP technique with better execution time. For example with $n=100$, the LP techniques provides $94.30 \%$ coverage, and the noncontiguous heuristic algorithm achieves $87.14 \%$ coverage. The LP technique requires 0.3 second, and the heuristic algorithm requires 0.17 second to solve the problem. In the noncontiguous heuristic algorithm, the sum of distance traversed by all sensors is $6.75 \%$ higher than that of the LP technique. 


\section{Chapter 5}

\section{Conclusion and Future Research}

In this work, we explored the problem of computing final positions of the sensors to form a line segment barrier. However finding an optimal barrier coverage with minimal movement is challenging. We formulate the problem as a LP framework to find the optimal relocations with a guaranteed level of coverage. The proposed method utilizes the sensor's mobility to improve the coverage. We evaluated the performance of the barrier coverage and showed that proposed methods improved the coverage. We also proved the accuracy of the algorithms presented in [21], [22] by comparing with the LP techniques. All previous work on minimal movement barrier coverage considered only static sensing range. We presented solution for both static and variable sensing range.

\subsection{Summary of the work}

Here we list the insights gained from this work that can be useful to design an effective line segment barrier.

- Although deterministic sensor placement strategy greatly minimizes the number of sensors, random deployment is more feasible due to large geographic area and many other practical constraints.

- Unlike static sensors, the mobile sensors can organize and appropriately reposition themselves in the network and achieve a higher degree of coverage and 
connectivity.

- The distributed approach only uses local information to find a solution, and therefore scales to network size. However the number of messages required per sensor could be very high. Also decision made with local information is always suboptimal. The central system can make optimal decisions with global information.

- The improvement of the coverage in contiguous approach is equal to that of noncontiguous approach for both 'MinSum' or 'MinMax' variants. The sum of distance traversed is higher in contiguous approach as compared to that of the noncontiguous approach irrespective of 'MinSum' and 'MinMax' variants. This is due to the fact that sensors in contiguous case have to move larger distance to ensure the contiguity requirement.

- The sum of distances is higher in 'MinMax' variant when compared to that of the 'MinSum' variant. However the maximum distance traversed by a sensor among all sensors is higher in 'MinSum' case than that of the 'MinMax' case.

- The variable range contiguous LP technique (both 'MinSum' and 'MinMax' approaches) is infeasible almost in all cases. This is due to the fact that most of the sensors in this technique have to traverse a long way to ensure the contiguity. In doing so, their sensing ranges reduce significantly. This leads to violations of constraints and the LP solution appears infeasible.

- The proposed heuristic algorithm can compute the final positions of the sensors with significantly faster run time when compared to that of the LP technique. 
The performance of heuristic algorithm is also close to the optimal method in terms of coverage measure and sum of distance traversed.

\subsection{Open problem and future work}

In this thesis, we presented many important findings that are useful to select coverage type and to improve the detection probability in an energy efficient way. There are still few open problems that need to be addressed to make MSN applications a complete success. We consider that the sensing range reduces linearly with the distance traversed. Since the performance depends on the sensing range significantly, the accuracy of sensing range model demands more investigations.

In our model, all sensors are able to move. Few researchers suggested that static sensors in a hybrid network can assist the mobile sensors to reduce the movements. Our method only provides coverage for a line segment. The insight gained from this study can be extended to protect a planar region in an energy efficient way. The optimal solution of such a problem in a three dimensional network is even more challenging. Few examples of three dimensional MSN are underwater marine life monitoring, coral reef monitoring, and atmospheric weather forecasting. Our proposed method is centralized in nature where we assume that all sensors can communicate with the central system. There is scope to investigate the distributed version of the proposed method. The communication model is very important as in the distributed approach, sensors need to communicate with local neighbors for collecting information. There are a few other issues such as connectivity architectures and security and management that need to be resolved so as to close the gap between technology and application. 


\title{
List of Symbols
}

\author{
$n \quad$ Number of Sensors \\ $L \quad$ Length of the Line \\ $S_{i} \quad$ Sensor $i$ \\ $r_{i} \quad$ Sensing range of the $i$-the sensor \\ $R \quad$ The sum of the sensing range \\ $I\left(S_{i}, x_{i}\right) \quad$ Closed interval of a line covered by sensor $S_{i}$ \\ $x_{i} \quad$ Initial position of $i$-th sensor \\ $y_{i} \quad$ Final position of $i$-th sensor \\ $d_{i} \quad$ Distance moved from initial $x_{i}$ to final position $y_{i}$ \\ $g_{i} \quad$ Gap $i$ \\ $o_{i} \quad$ Overlap $i$ \\ $\tau$ The proportional constant
}




\section{Abbreviations}

LP Linear Programming

MSN Mobile Sensor Network

WSN Wireless Sensor Network 


\section{References}

[1] J. S. Wilson, Sensor Technology Handbook. Amsterdam: Elsevier, 2005.

[2] G. Werner-Allen, K. Lorincz, M. Ruiz, O. Marcillo, J. Johnson, J. Lees, and M. Welsh, "Deploying a wireless sensor network on an active volcano," IEEE Internet Comput., vol. 10, no. 2, pp. 18-25, Mar. 2006.

[3] T. Naumowicz, R. Freeman, H. Kirk, B. Dean, M. Calsyn, A. Liers, A. Braendle, T. Guilford, and J. Schiller, "Wireless sensor network for habitat monitoring on skomer island," in IEEE Conf. on Local Computer Networks (LCN), Oct. 2010, pp. 882-889.

[4] A. Mainwaring, D. Culler, J. Polastre, R. Szewczyk, and J. Anderson, "Wireless sensor networks for habitat monitoring," in Proc. ACM Int. Workshop on Wireless Sensor Networks and Applic. (WSNA), 2002, pp. 88-97.

[5] C.-Y. Chong and S. P. Kumar, "Sensor networks: evolution, opportunities, and challenges," Proc. of the IEEE, vol. 91, no. 8, pp. 1247-1256, Aug. 2003.

[6] A. Arora, P. Dutta, S. Bapat, V. Kulathumani, H. Zhang, V. Naik, V. Mittal, H. Cao, M. Demirbas, M. Gouda, Y. Choi, T. Herman, S. Kulkarni, U. Arumugam, M. Nesterenko, A. Vora, and M. Miyashita, "A line in the sand: a wireless sensor network for target detection, classification, and tracking," $J$. Computer Netw., vol. 46, no. 5, pp. 605-634, 2004. 
[7] G. Simon, M. Maróti, A. Lédeczi, G. Balogh, B. Kusy, A. Nádas, G. Pap, J. Sallai, and K. Frampton, "Sensor network-based countersniper system," in Proc. International Conf. on Embedded Networked Sensor Syst. (SenSys). USA: ACM, 2004, pp. 1-12.

[8] G. Z. Yang, Body Sensor Networks, Berlin, 2006.

[9] K. Lorincz, D. J. Malan, T. R. F. Fulford-Jones, A. Nawoj, A. Clavel, V. Shnayder, G. Mainland, M. Welsh, and S. Moulton, "Sensor networks for emergency response: challenges and opportunities," IEEE Pervasive Comput., vol. 3, no. 4, pp. 16-23, Oct. 2004.

[10] K. Sohraby, D. Minoli, and T. Znati, Wireless Sensor Networks: Technology, Protocols, and Applications. Wiley-Interscience, 2007.

[11] P. Balister, B. Bollobas, A. Sarkar, and S. Kumar, "Reliable density estimates for coverage and connectivity in thin strips of finite length," in Proc. ACM Int. Conf. on Mobile Comput. and Netw. (MobiCom). ACM, 2007, pp. 75-86.

[12] A. Chen, T. H. Lai, and D. Xuan, "Measuring and guaranteeing quality of barrier-coverage in wireless sensor networks," in Proc. ACM Int. Symp. on Mobile Ad Hoc Netw. and Comput. (MobiHoc), 2008, pp. 421-430.

[13] G. Yang and D. Qiao, "Barrier information coverage with wireless sensors," in IEEE Infocom, Apr. 2009, pp. 918-926.

[14] S. Kumar, T. H. Lai, and A. Arora, "Barrier coverage with wireless sensors," Proc. of ACM MOBICOM, Aug. 2005. 
[15] B. Liu, O. Dousse, J. Wang, and A. Saipulla, "Strong barrier coverage of wireless sensor networks," Proc. ACM Int. Symp. Mobile Ad Hoc Netw. Comput. (MobiHoc), 2008.

[16] B. Bhattacharya, B. Burmester, Y. Hu, E. Kranakis, Q. Shi, and A. Wiese, "Optimal movement of mobile sensors for barrier coverage of a planar region," in Proc. Int. Conf. on Combinat. Opt. and Applic. (COCOA). Springer-Verlag, 2008, pp. 103-115.

[17] B. Bhattacharya, M. Burmester, Y. Hu, E. Kranakis, Q. Shi, and A. Wiese, "Optimal movement of mobile sensors for barrier coverage of a planar region," J. Theoretical Computer Science, vol. 410, no. 52, pp. 5515-5528, 2009.

[18] X. Tan and G. Wu, New Algorithms for Barrier Coverage with Mobile Sensors, ser. Frontiers in Algorithmics. Springer Berlin Heidelberg, 2010, vol. 6213, pp. $327-338$.

[19] A. Saipulla, B. Liu, G. Xing, X. Fu, and J. Wang, "Barrier coverage with sensors of limited mobility," in Proc. ACM Int. Symp. on Mobile Ad Hoc Netw. and Comput. (MobiHoc), 2010, pp. 201-210.

[20] P. W. Shor and J. E. Yukich, "Minimax grid matching and empirical measures," J. The Annals of Probability, vol. 19, no. 3, pp. 1338-1348, 1991.

[21] J. Czyzowicz, E. Kranakis, D. Krizanc, I. Lambadaris, L. Narayanan, J. Opatrny, L. Stacho, J. Urrutia, and M. Yazdani, On Minimizing the Maximum Sensor Movement for Barrier Coverage of a Line Segment, ser. Ad-Hoc, Mobile and Wireless Networks. Springer Berlin Heidelberg, 2009, pp. 194-212. 
[22] — - On Minimizing the Sum of Sensor Movements for Barrier Coverage of a Line Segment, ser. Ad-Hoc, Mobile and Wireless Networks. Springer Berlin Heidelberg, 2010, pp. 29-42.

[23] S. Kumar, "Foundations of coverage in wireless sensor networks," Ph.D. dissertation, Ohio State University, OH, USA, 2006.

[24] T. Clouqueur, V. Phipatanasuphorn, P. Ramanathan, and K. K. Saluja, "Sensor deployment strategy for target detection," in Proc. ACM Int. Workshop on Wireless Sensor Netw. and Applic. (WSNA), 2002, pp. 42-48.

[25] S. S. Dhillon and K. Chakrabarty, "Sensor placement for effective coverage and surveillance in distributed sensor networks," in IEEE Wireless Communications and Networking (WCNC), Mar. 2003, pp. 1609-1614.

[26] D. Pompili, T. Melodia, and I. F. Akyildiz, "Deployment analysis in underwater acoustic wireless sensor networks," in Proc. ACM Int. Workshop on Underwater Netw. (WUWNet). New York, NY, USA: ACM, 2006, pp. 48-55.

[27] E. S. Biagioni and G. Sasaki, "Wireless sensor placement for reliable and efficient data collection," in Proc. Int. Conf. on Syst. Sciences, Jan. 2003, pp. 1-10.

[28] M. Mehrandish, L. Narayanan, and J. Opatrny, "Minimizing the number of sensors moved on line barriers," in IEEE Wireless Communications and Networking Conference (WCNC), Mar. 2011, pp. 653-658.

[29] B. Liu and D. Towsley, "A study of the coverage of large-scale sensor networks," in IEEE Int. Conf. Mobile Ad-hoc and Sensor Syst., 2004, pp. 475-483. 
[30] C. F. Huang and Y. C. Tseng, "A survey of solutions to the coverage problems in wireless sensor networks," J. Internet Technol., vol. 6, no. 1, pp. 1-8, 2005.

[31] E. Garcia and P. G. de Santos, "Hybrid deliberative/reactive control of a scanning system for landmine detection," Robot. Auton. Syst., vol. 55, no. 6, pp. 490-497, Jun. 2007.

[32] G. Wang, G. Cao, and T. L. Porta, "Movement-assisted sensor deployment," IEEE Trans. Mobile Comput., vol. 5, no. 6, pp. 640-652, 2006.

[33] F. Aurenhammer, "Voronoi diagrams - a survey of a fundamental geometric data structure," ACM Comput. Surv., vol. 23, no. 3, pp. 345-405, Sep. 1991.

[34] H. Zhang and J. Hou, "Maintaining sensing coverage and connectivity in large sensor networks," Int. J. Ad Hoc and Sensor Wireless Netw., vol. 1, no. 1-2, pp. $89-123,2005$.

[35] B. Liu, P. Brass, O. Dousse, P. Nain, and D. Towsley, "Mobility improves coverage of sensor networks," in Proc. ACM Int. Symp. on Mobile Ad Hoc Netw. and Comput. (MobiHoc), 2005, pp. 300-308.

[36] C. F. Huang and Y.-C. Tseng, "The coverage problem in a wireless sensor network," in Proc. ACM Int. Conf. on Wireless Sensor Netw. and Applic., Sept. 2003, pp. 115-121.

[37] A. Saipulla, C. Westphal, B. Liu, and J. Wang, "Barrier coverage of line-based deployed wireless sensor networks," IEEE Infocom, pp. 127-135, 2009. 
[38] S. Kumar, T. H. Lai, M. E. Posner, and P. Sinha, "Optimal sleep wakeup algorithms for barriers of wireless sensors." IEEE Int. Conf. on Broadband Commun., Netw., and Syst., pp. 327-336, 2007.

[39] A. Ghosh and S. K. Das, "Coverage and connectivity issues in wireless sensor networks: A survey," J. Pervasive and Mobile Comput., vol. 4, no. 3, pp. 303$334,2008$.

[40] S. Meguerdichian, F. Koushanfar, M. Potkonjak, and M. B. Srivastava, "Coverage problems in wireless ad-hoc sensor networks," in IEEE Infocom, 2001, pp. 1380-1387.

[41] S. Meguerdichian, F. Koushanfar, G. Qu, and M. Potkonjak, "Exposure in wireless ad-hoc sensor networks," in Proc. Int. Conf. on Mobile Comput. and Netw. (MobiCom). ACM, 2001, pp. 139-150.

[42] S. Kumar, T. H. Lai, and J. Balogh, "On $k$-coverage in a mostly sleeping sensor network," in Proc. Int. Conf. on Mobile Comput. and Netw. (MobiCom). ACM, 2004, pp. 144-158.

[43] V. Raghunathan, C. Schurgers, S. Park, and M. B. Srivastava, "Energy-aware wireless microsensor networks," IEEE Signal Proc. Magazine, vol. 19, no. 2, pp. 40-50, Mar. 2002.

[44] M. Cardei, M. T. Thai, Y. Li, and W. Wu, "Energy-efficient target coverage in wireless sensor networks," in IEEE Infocom, Mar. 2005, pp. 1976-1984.

[45] S. Meguerdichian and M. Potkonjak, "Low power 0/1 coverage and scheduling techniques in sensor networks," UCLA Technical Reports 030001, 2003. 
[46] L. Kong, X. Liu, Z. Li, and M. Y. Wu, "Automatic barrier coverage formation with mobile sensor networks," IEEE International Conference on Communication (ICC), pp. 1-5, 2010.

[47] A. Chen, S. Kumar, and T. H. Lai, "Designing localized algorithms for barrier coverage," Proc. ACM Int. Conf. on Mobile Comput. and Netw., pp. 63-74, 2007.

[48] T. M. Cheng and A. V. Savkin, "A distributed self deployment algorithm for the coverage of mobile wireless sensor networks," IEEE Commun. Lett., vol. 13, no. 11, pp. $877-879,2009$.

[49] C. Shen, W. Cheng, X. Liao, and S. Peng, "Barrier coverage with mobile sensors," Proc. Int. Symp. Parallel Architectures, Algorithms, and Netw., pp. 99-104, May 2008.

[50] M. Li, X. Sun, and Y. Zhao, Minimum-Cost Linear Coverage by Sensors with Adjustable Ranges, ser. Wireless Algorithms, Systems, and Applications, 2011, vol. 6843, pp. $25-35$.

[51] A. Lvov and F.-L. Heng, "A graph based simplex method for the integer minimum perturbation problem with sum and difference constraints," in Proc. ACM Great Lakes Symp. on VLSI, 2004, pp. 67-72.

[52] D. F. Shanno and R. L. Weil, "Linear programming with absolute-value functionals," Operations research, vol. 19, no. 1, pp. 120-124, 1971.

[53] I. Corporation, "Ibm ilog cplex optimizer," 2012. [Online]. Available: http://www.ibm.com 
[54] G. Wang, M. J. Irwin, H. Fu, P. Berman, W. Zhang, and T. L. Porta, "Optimizing sensor movement planning for energy efficiency," ACM Trans. Sen. Netw., vol. 7, no. 4, pp. 1-17, Feb. 2011. 